\title{
亚硝基-烯(Nitroso-ene)反应的研究进展
}

\author{
黄莎华*,a,b 霍华兴 ${ }^{b}$ 李文华 $b$ 洪 然*,b \\ $\left({ }^{a}\right.$ 上海应用技术学院 上海 201418) \\ ( ${ }^{b}$ 中国科学院上海有机化学研究所 上海 200032)
}

\begin{abstract}
摘要 烯丙基胺类化合物是有机化学中的重要合成中间体，亚硝基-烯(nitroso-ene)反应是合成这类化合物最为高效的 方法之一. 在一些天然产物和药物分子的全合成中，亚硝基一烯反应也往往作为关键步骤. 这篇综述主要介绍了亚硝 基-烯反应的发展、应用以及机理研究方面的进展.

关键词 烯丙基胺; 亚硝基化合物; 亚硝基-烯(nitroso-ene)反应; 天然产物合成
\end{abstract}

\section{Research Progress on Nitroso-ene Reaction}

\author{
Huang, Shahua ${ }^{*, a, b}$ Huo, Huaxing ${ }^{b} \quad$ Li, Wenhua ${ }^{b} \quad$ Hong, Ran ${ }^{*, b}$ \\ ( ${ }^{a}$ Shanghai Institute of Technology, Shanghai 201418) \\ $\left({ }^{b}\right.$ Shanghai Institute of Organic Chemistry, Chinese Academy of Sciences, Shanghai 200032)
}

\begin{abstract}
Nitroso-ene reaction is one of the most efficient methods to construct allylamines which are the versatile building block in synthetic organic chemistry. The reaction has been embedded as the key step in the total synthesis of natural products and pharmaceuticals. This review focuses on the development, application and mechanistic study of nitroso-ene reaction.

Keywords allylamine; nitroso compound; nitroso-ene reaction; natural product synthesis
\end{abstract}

\section{1 背景介绍}

早在 1874 年科学家们便首次合成出亚硝基化合物 并进行了鉴定 ${ }^{[1]}$, 但是多年来其反应活性特别是在有机 合成中的应用并没有受到足够的重视. 近三十多年来, 人们对亚硝基化合物的结构及性质有了较为深入的认 识和了解 ${ }^{[2]}$. 尽管如此, 在基础有机化学的教学中, 仅 是在还原芳香硝基化合物得到亚硝基苯中间还原产物 中有所提及, 并没有对亚硝基化合物的性质和反应类型 进行详细的介绍.

氮氧化合物在生物体内也较为常见，它们通常以羟 胺、硝基以及亚硝基形式存在, 且均含有重要的生理活 性, 如抗癌药 calicheamicin 及抗菌素氯需素等 ${ }^{[3]} .2010$ 年, Kersten 和 Dorrestein ${ }^{[4]}$ 通过对含有亚硝基官能团天 然产物 4,3-HNBAm 生物机制的研究, 初步揭示了亚硝 基化合物在生物体内的合成途径, 即利用氧化酶 $\mathrm{NspF}$ 将胺类化合物氧化成亚硝基类产物.

亚硝基化合物是一类具有高活性的反应中间体，能
够进行多种反应从而得到不同含有氮氧官能团化的产 物 ${ }^{[5,6]}$. 例如亚硝基化合物可以通过杂 Diels-Alder 反 应 $^{[7]}$ 、Aldol 加成反应 ${ }^{[8]}$ 以及 ene 反应 ${ }^{[9]}$ 等来分别得到含 有 $\delta$-氨基醇、烯丙基胺、羰基 $\alpha$ 位氨化或羟胺化等在药 物分子和天然产物中常见的结构单元，同时它们还是有 机合成化学中极为重要的中间体 ${ }^{[10]}$. 但在反应过程中, 由于亚硝基化合物非常高的反应活性，往往导致多种副 反应的发生，从而限制了其在有机合成化学中的发展及 应用.

本篇综述将主要介绍亚硝基-烯反应这一最近才受 到重视的领域，涉及亚硝基-烯反应的发展、应用及机理 研究，以期推动这一领域的发展.

\section{1 亚硝基化合物的反应性}

含有亚硝基官能团的化合物通常具有很强的亲电 性质，可以进行一系列的反应，如加成反应、异构化、 氧化及还原反应 ${ }^{[5,6]}$. 从 Scheme 1 中可以看出, 作为亲 电试剂，它能够与烯醇化物 ${ }^{[1]}$ (路线 a)、胺类化合

\footnotetext{
*E-mail: shahua@sit.edu.cn; rhong@sioc.ac.cn

Received July 18, 2012; revised August 8, 2012; published online August 10, 2012.

Project supported by the Young Teachers Program of Universities in Shanghai (No. yyy11015).

上海高校青年教师培养资助计划(No. yyy11015)资助项目.
} 
物 $^{[12]}$ (路线 b) 和格氏试剂 ${ }^{[13]}$ (路线 c)发生加成反应. 当与 重氮甲烷反应时, 可以得到硝酮类产物 $\mathbf{4}^{[14]}$ (路线 $\mathrm{d}$ ). 亚 硝基化合物还是很好的自由基捕获剂 ${ }^{[15]}$ (路线 e). 同时, 它还能发生 $[2+2]^{[16]}$ (路线 f)、 $[4+2]^{[17]}$ (路线 g) 环加成反 应以及烯(ene)反应 ${ }^{[18]}$ (路线 $\left.h\right)$.

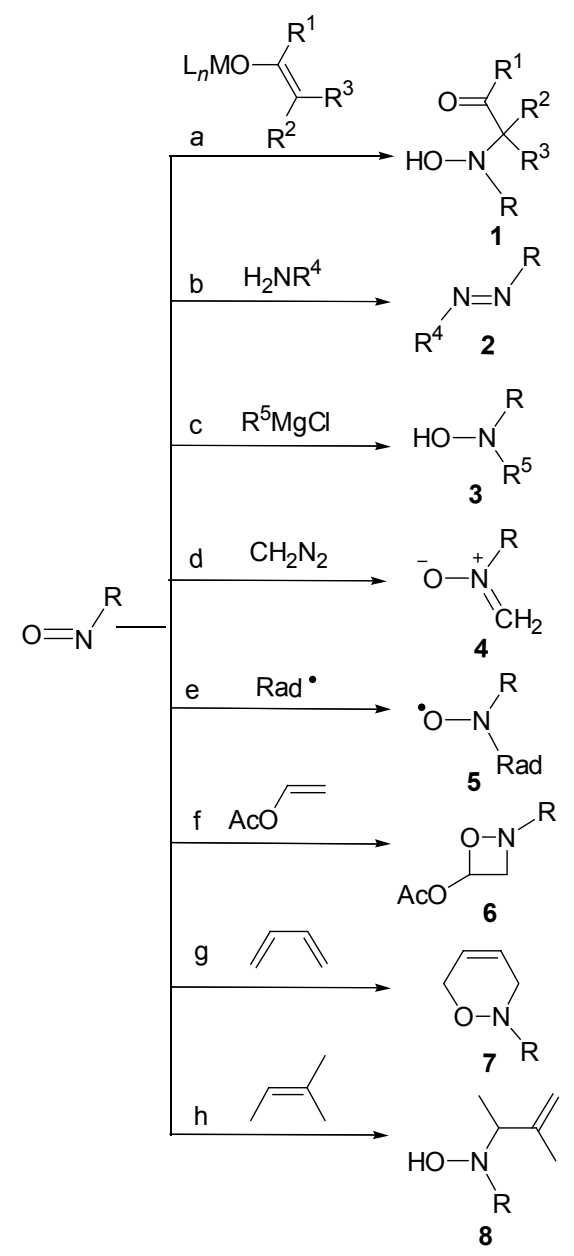

Scheme 1

亚硝基化合物具有如此高的反应活性以及能进行 多种反应主要是因为其较低的 LUMO 轨道能量, 容易 接受电子, 使其成为很好的亲电试剂. 同时, 由于其氮 上孤对电子占据较高的 HOMO 轨道能量, 使它还具有 一定的亲核性. 因此, 在一定条件下亚硝基化合物能够 发生二聚(Scheme 2), 并存在顺式和反式两种构型. 在 溶液中它们以平衡形式存在, 其比例的大小主要依赖于 取代基 $\mathrm{R}^{[19]}$. 亚硝基化合物的这一性质一定程度上也限 制了其在有机合成中的应用.<smiles>[R]N=NC=CC</smiles>

Scheme 2

\section{2 亚硝基化合物具有代表性的反应}

在亚硝基化合物可以进行的众多反应类型中, 以杂 Diels-Alder 反应和 aldol 反应是不对称反应研究的重点, 得到了大家的广泛关注. 因为有大量的文献涉及这些领 域的研究工作, 可以参考相关的综述 ${ }^{[20]}$, 这里只做简单 的介绍.

\subsection{1 亚硝基化合物参与的杂 Diels-Alder 反应}

人们对亚硝基化合物作为亲双烯体参与的杂 Diels-Alder 环加成反应进行了深入研究, 并常将其作为 重要反应用于天然产物全合成和药物分子的合成中. 这 是因为反应不仅能取得优异的区域选择性和立体选择 性 $^{[17 \mathrm{~d}]}$, 亚硝基化合物的环加成产物 12 可以经过简单的 转化得到一系列多样化的官能团化产物, 如吡咯烷衍生 物 13，1,4-氨基醇类化合物 14 以及氮杂糖 15 等 ${ }^{[21]}$ (Scheme 3).

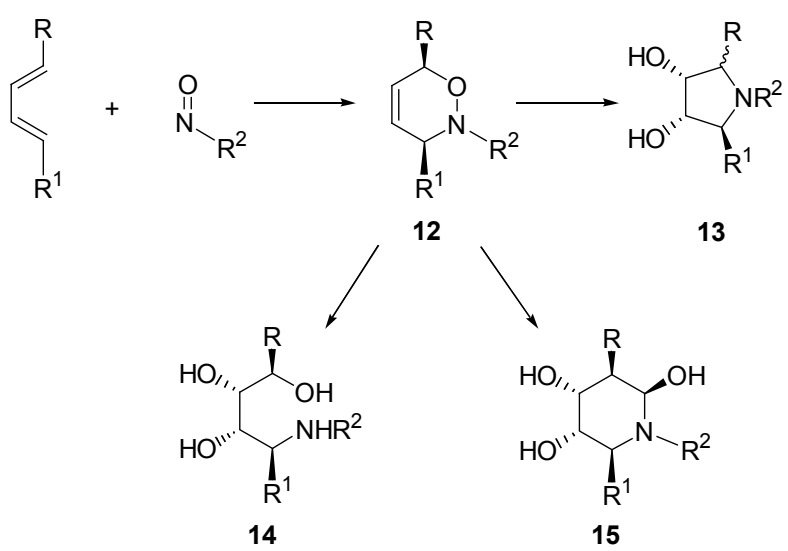

Scheme 3

\subsection{2 亚硝基类 aldol 反应}

光学纯的 $\alpha$-氨基酸是合成手性试剂、催化剂和天然 产物的重要起始原料. 亚硝基 aldol 反应就是制备这一 类 $\alpha$-氨基酸的有效方法之一 ${ }^{[22]}$, 同时它还能得到其他 手性的羰基 $\alpha$ 位氨化或羟胺化类化合物.

与经典的羰基 aldol 反应不同，亚硝基类 aldol 反应 会有烯醇化合物进攻氧或氮两条途径并得到两种不同 的产物 16 和 17 (Scheme 4). 反应进行的途径主要取决 于烯醇化合物的选择和路易斯酸的使用 ${ }^{[23]}$. 通常情况 下，金属如锌盐催化剂会与亚硝基化合物中的氧原子配 位并极化氮氧共价键从而使烯醇锂盐进攻氮原子, 而当 使用有机小分子催化剂时烯醇硅醚则会主要进攻氧原 子.

最近几年来，人们对不对称亚硝基类 aldol 反应进 行了深入的研究. 1992 年, Oppolzer 小组 ${ }^{[22]}$ 报道了利用 樟脑磺酸衍生物作为手性辅基来进行不对称亚硝基类 aldol 反应并最终得到光学纯的 $\alpha$-氨基酸. 之后其他研 究小组发展了一系列使用手性有机小分子或金属催化 
$\underbrace{\mathrm{O}}_{\mathrm{R}^{3}} \mathrm{P}_{\mathrm{R}}^{\mathrm{OH}}{ }_{\mathrm{ZnCl}_{2}}^{\mathrm{R}^{2}=\mathrm{Li}}$

16

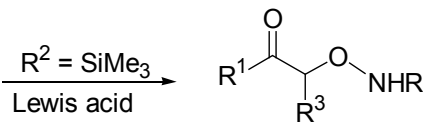

17

\section{Scheme 4}

剂来催化不对称亚硝基类 aldol 反应, 均可以得到很高 的对映选择性 ${ }^{[24]}$ (Scheme 5).

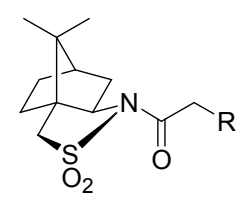

18

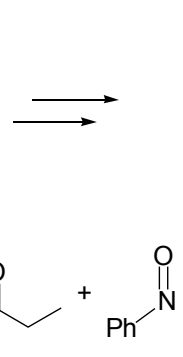

20

21

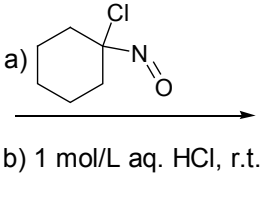

b) $1 \mathrm{~mol} / \mathrm{L}$ aq. $\mathrm{HCl}$, r.t

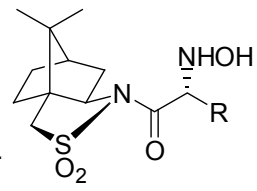

19<smiles>[R]C(N)C(=O)O</smiles>

20

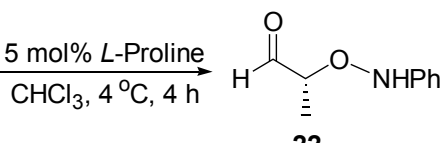
$88 \%, 97 \%$ ee<smiles>CCCCCCCCCCCCCCCCC</smiles>
(R)-BINAP. 2 AgOTf (4 $\mathrm{mol} \%)$<smiles>O=C1CCCCC1N(O)c1ccccc1</smiles>

24 $95 \%,>99 \%$ ee
Scheme 5

\section{2 亚硝基-烯反应}

\section{1 亚硝基-烯反应的介绍}

烯(ene) 反应是有机合成中一类简单实用的化学反 应，根据亲电试剂的不同可合成官能团多样化的产 物 $^{[25]}$. 从反应的过渡态考虑, 烯反应也被认为是一类 $\pi_{2 s}+\pi_{2 s}+\sigma_{2 s}$ 反应 ${ }^{[26]}$ (Scheme 6). 当亲电试剂为亚硝基 化合物时, 即称为亚硝基-烯反应, 生成的产物是烯丙 基胺类化合物.

从通式中可以看出，亚硝基一烯反应能够得到烯丙 基胺类化合物. 这一类化合物是天然产物及有机合成中 重要的结构单元, 可以用于合成生物碱、 $\alpha$-和 $\beta$-氨基酸 以及碳水化合物衍生物等 ${ }^{[27]}$. 合成烯丙基胺类化合物

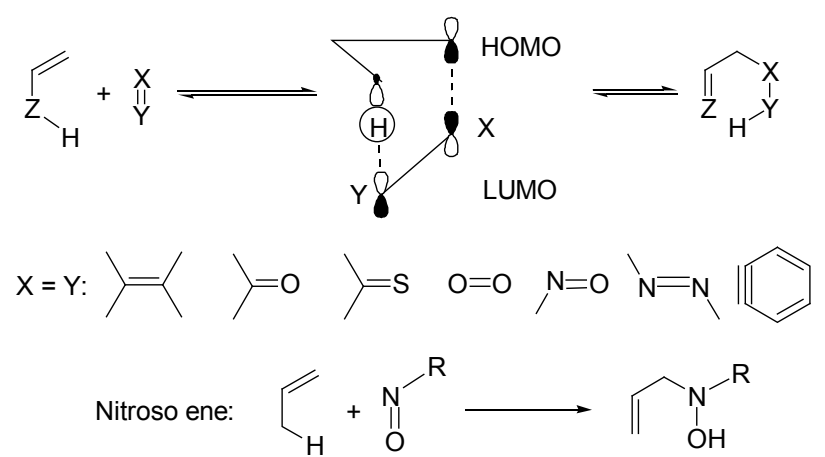

Scheme 6

通常有三种策略: (1)烯丙位亲核取代, 如 Mitsonobu 反 应和 Gabriel 反应; (2)直接用烯基金属试剂对亚胺的加 成；(3)对含有双键底物的烯丙基胺化反应，如过渡金属 催化的烯丙位直接胺化等. 亚硝基一烯反应是可以在不 用过渡金属催化条件下直接对烯烃的 $\alpha$ 位进行胺化反 应. 很显然，这一策略避免使用昂贵的试剂，可以从简 单的含双键底物直接转化成烯丙基胺类及其官能团化 的产物 ${ }^{[18 b]}$, 有很好的应用前景.

\section{1 .1 亚硝基一烯反应中的副反应}

早在 1965 年, Haszeldine 小组 ${ }^{[28]}$ 便发现了亚硝基烯反应，但直至近些年才得到人们的关注. 1959 年 Luttke 小组 ${ }^{[29]}$ 通过理论分析对烷基亚硝基化合物的二 聚及解聚进行了研究, 发现解聚的活化能并不高, 比如 2-甲基-2-亚硝基戊酮在苯中解聚的活化能为 $100 \mathrm{~kJ}$ • $\mathrm{mol}^{-1}$. 但对于酰基亚硝基类化合物 25 发生二聚后会进 一步分解得到酸酐 26 及 $\mathrm{N}_{2} \mathrm{O}$ 气体从而无法进行亚硝 基一烯反应 ${ }^{[30]}$ (Scheme 7).
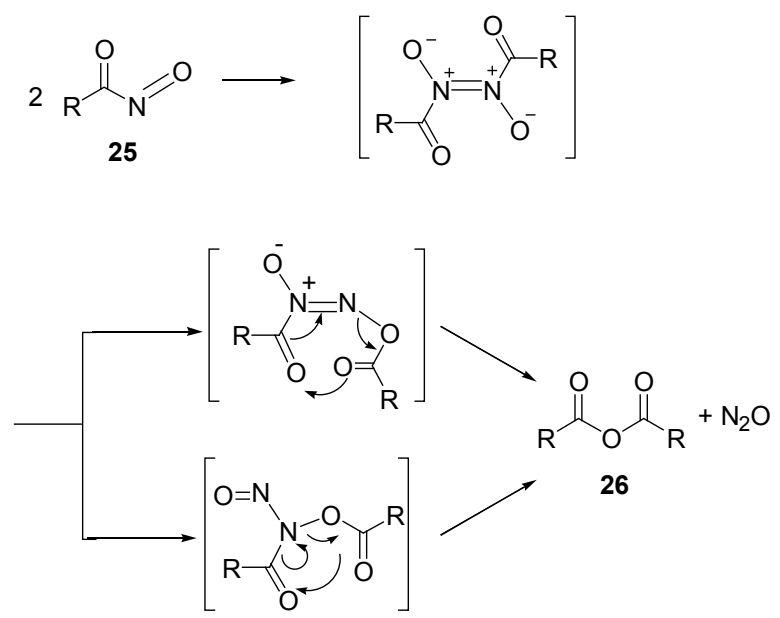

Scheme 7

人们发现当连有吸电子取代基时，亚硝基的亲电性 及反应活性会增加. 例如酰基亚硝基化合物和 $\alpha$-卤代亚 硝基化合物(Scheme 8). 尤其是酰基亚硝基化合物, 由 
于其较低的活化能和较小的 HOMO-LUMO 轨道能级 差, 跟其他亚硝基化合物相比它有着更高的活性 ${ }^{[31]}$. 而 芳香类取代的亚硝基化合物则相对稳定, 如亚硝基苯甚 至可以直接购得, 但其反应活性相对较低.

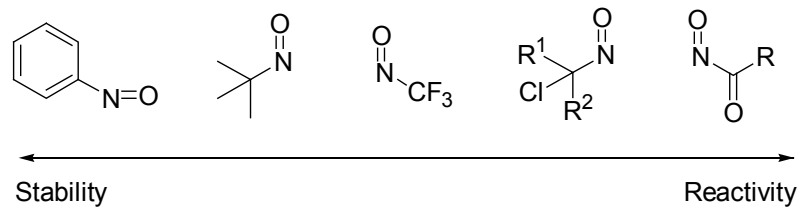

\section{Scheme 8}

当使用亚硝基苯进行亚硝基-烯反应时, 发现其产 物羟胺化合物 27 稳定性较差, 会和体系中存在的氧化 剂继续发生原位氧化、分解和还原反应等, 从而转化成 相应的硝酮 28、氮氧化合物 30、亚胺 29、烯丙基胺 33 及氧代偶氮类化合物 $\mathbf{3 2}^{[32]}$ (Scheme 9), 使得反应体系变 得相当复杂. 这一特殊的反应特性极大地增加了亚硝 基-烯反应的控制难度，限制了其在有机化学中的应用 和发展.

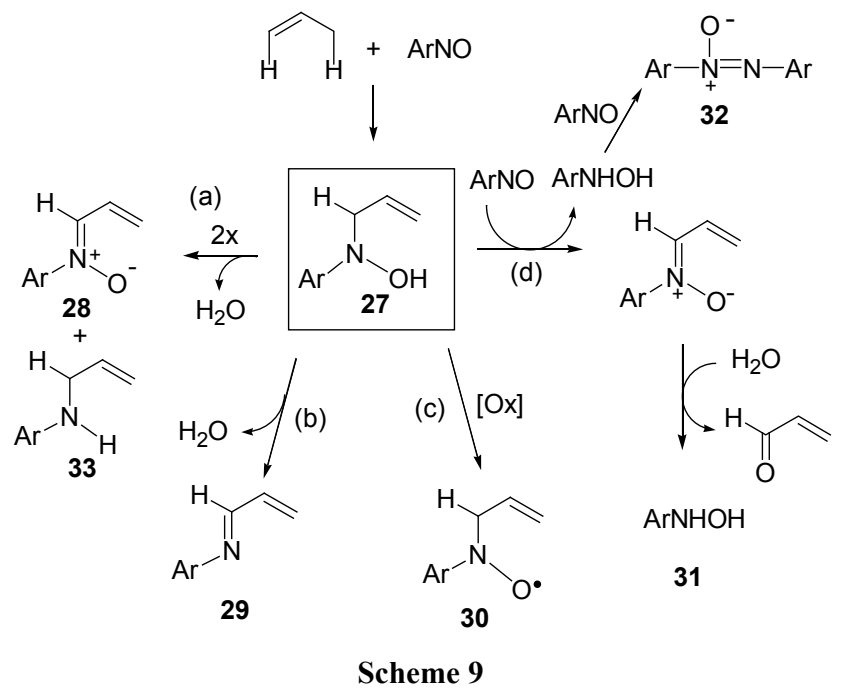

\section{2 亚硝基-烯反应的发展}

当缺电子的亚硝基化合物作为亲电试剂进行亚硝 基-烯反应时, 可以较好地抑制其产物会继续发生原位 反应的问题. 因为缺电子的产物较难被进一步氧化并发 生其他副反应. 但是缺电子的亚硝基化合物如酰基亚硝 基化合物极易发生二聚, 所以人们设计了将亚硝基化合 物的合成及亚硝基-烯反应采取 “两步一锅法” 来实现, 避免因原料在体系中积聚而发生二聚. 因此多年来化学 家们在亚硝基中间体制备方法的改进及亚硝基化合物 底物选择的发展做了很多贡献.

\subsection{1 亚硝基中间体制备方法的发展}

通常现场制备高活性的酰基类亚硝基中间体的方
法有 5 种 ${ }^{[33]}$ : (1)逆 Diels-Alder 反应; (2)硝基卡宾化物重 排; (3)用 $N$-甲基氧化吗啡啉(NMO)氧化氰基氧化物; (4) 光解氧代噁二唑类化合物. (5)氧化酰基羟胺化合物(见 Scheme 10). 显然最后一种方法更直接也具有原子经济 性，但在常规的氧化条件下，亚硝基化合物很难稳定存 在，也会因自身聚合裂解而消耗 ${ }^{[34]}$.

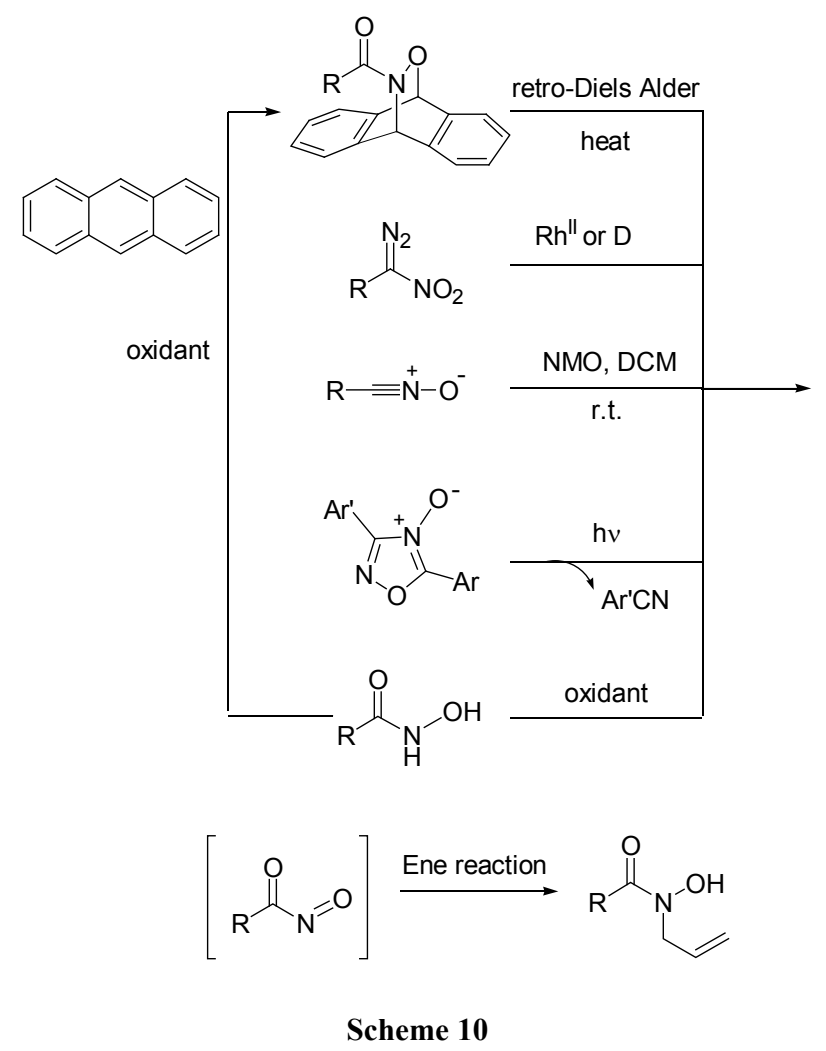

2.2.1.1 用逆 Diels-Alder 反应来制备亚硝基中间体

为了防止副反应的发生和除去氧化剂对后续反应 的影响, Kirby ${ }^{[35]}$ 发展了 $[4+2]$ 环加成反应现场捕获亚硝 基中间体的方法. 其环加成产物在加热至大约 $100{ }^{\circ} \mathrm{C}$ 的情况下便可进行逆 Diels-Alder 反应释放出亚硝基化 合物，不过他们只是利用这个方法来尝试酰基亚硝基化 合物后续的杂 Diels-Alder 反应.

1981 年, Keck 小组 ${ }^{[36]}$ 首次用这种逆 Diels-Alder 反 应策略制备亚硝基中间体方法来进行亚硝基-烯反应. 在分子间亚烯反应的尝试中，发现使用环内、环外烯烃 或是二取代、三取代烯烃能以 $83 \% \sim 95 \%$ 的较高产率获 得加成产物. 而对于分子内亚硝基-烯反应，不仅产率 有所提高, 同时反应时间也缩短至 30 至 $40 \mathrm{~min}$ (Scheme 11).

Ensley 小组对最简单的亚硝酰和亚硝基甲酰的烯 反应有很大的兴趣. 他们用无水液氨脱除环加成产物 38 中的甲酰基, 再加热释放出亚硝酰中间体与双键底 物 40 进行亚硝基-烯反应, 得到目标产物 41 及半缩酮产 
<smiles>CC(=O)N1C(=O)C2(C)c3ccccc3C1(C)c1ccccc12</smiles>

34<smiles></smiles><smiles>[R]N(O)C(C)=O</smiles>

$3583 \% \sim 95 \%$<smiles>[Y]C1C=CC(=[R])CC1</smiles><smiles>C=C(C)C(C)C(CC)C(C)C(=C)C</smiles><smiles>[X]C(/C=C/C)CC(=O)N1C(C)(C)c2ccccc2C1(C)OC(=O)C(C)(C)C</smiles>

36

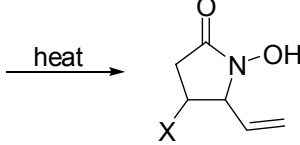

37
$\mathrm{X}=\mathrm{OSiR}_{3}, \mathrm{H}$

\section{Scheme 11}

物 42. 而当加成产物 38 直接与双键底物 40 反应时, 结 果却非常复杂, 还有不同区域选择性的产物 ${ }^{[37]}$ (Scheme 12).

2.2.1.2 用硝基卡宾化物重排的方法来制备酰基亚 硝基中间体

1969 年, Schollkopf 小组 ${ }^{[38]}$ 尝试硝基卡宾化物与 2,3-二甲基-2-丁烯反应，结果并没有得到预想的环丙烷 化产物, 而是分离得到将近 30\% 的硝基卡宾重排生成 酰基亚硝基中间体与 2,3-二甲基-2-丁烯进行烯反应的 产物(Scheme 13). 随后经过 Dailey 小组对硝基卡宾化物 的研究, 确定了其在加热或二价铑金属催化的条件下可 以重排生成酰基亚硝基中间体，并与蒽发生杂 Diels-Alder 反应，但只有与酯基或三氟甲基相连的硝基 卡宾化物才能得到较好结果 ${ }^{[39]}$.

2.2.1.3 用 $\mathrm{NMO}$ 氧化氰基氧化物的方法来制备酰基 亚硝基中间体

1998 年, Caramella 小组 ${ }^{[40]}$ 发展了用 $N$-甲基氧化吗 啡啉(NMO)氧化氰基氧化物 47 来制备亚硝基中间体, 而 NMO 并不会氧化烯产物 48. 尽管对于含有各种官能 团的酰基亚硝基底物均可以得到很好的结果，但当使用 较少取代的烯烃底物反应时, 氭基氧化物会直接发生 $[1,3]$-偶极环加成, 使得底物的适用范围受限(Scheme 14).
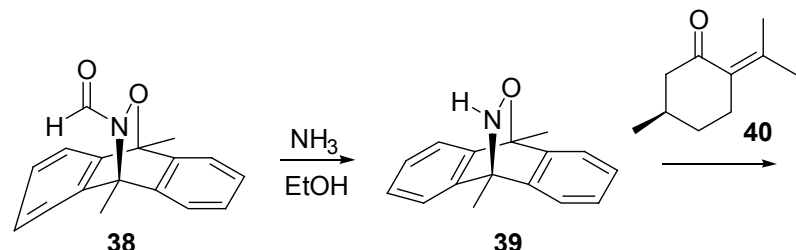

38

39<smiles>[Z14]C1C=C(C(C)(C)N(O)O)C(=O)C[C@@H]1C</smiles>

42

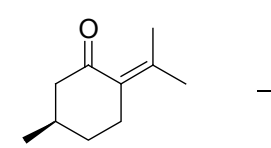

40

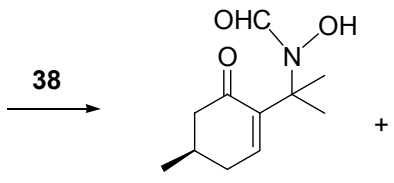

43

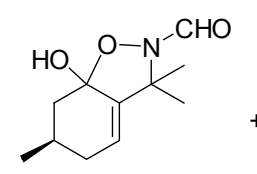

44<smiles>C=C(C)C1(N(C)O)CC[C@@H](C)CC1=O</smiles>

45

\section{Scheme 12}<smiles>CCOC(=O)C1([N+](=O)[O-])C(C)(C)C1(C)C</smiles><smiles>CC(C)=C(C)C(C)(C)C(C)(C)C</smiles>

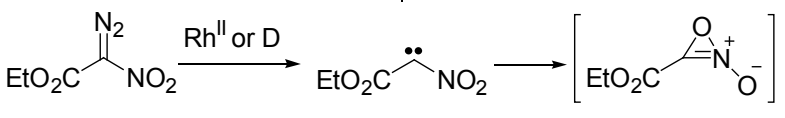<smiles>C=C(C)C(C)(C)N(O)C(=O)COC(=O)C(C)=C(C)C</smiles>

Scheme 13

2.2.1.4 光解氧代噁二唑类化合物来制备酰基亚硝 基中间体

为了解决 $\mathrm{NMO}$ 氧化氰基氧化物方法存在的弊端, Caramella 小组 ${ }^{[41]}$ 在次年发表了利用光解氧代噁二唑类 化合物 50 来制备酰基亚硝基中间体. 与之前的方法相 比，不仅能与四取代烯烃底物反应，获得高产率的加成 产物，对于较少取代的烯烃双键底物也获得有很好的结 果(Scheme 15).

以上讨论的三种方法通常都需要过量的烯烃底物, 而且亚硝基前体均需要预先合成，操作很不方便，同时 


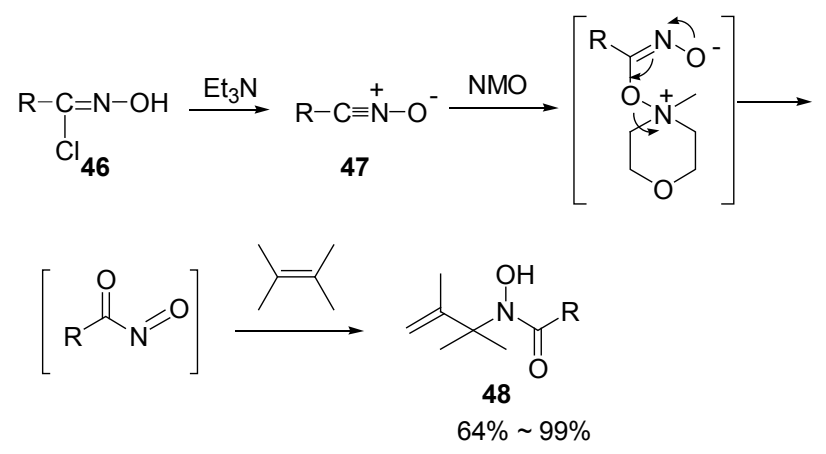

$\mathrm{R}=\mathrm{Ph}, p-\mathrm{ClC}_{6} \mathrm{H}_{4}, 2,4,6-\mathrm{Me}_{3} \mathrm{C}_{6} \mathrm{H}_{2}, p-\mathrm{MeC}_{6} \mathrm{H}_{4}, p-\mathrm{MeOC}_{6} \mathrm{H}_{4}, p-\mathrm{NO}_{2} \mathrm{C}_{6} \mathrm{H}_{4}$ $\mathrm{Me}, n-\mathrm{Pr}, \mathrm{Me}\left(\mathrm{CH}_{2}\right)_{5}, \mathrm{PhCH}_{2}, \mathrm{PhCH}=\mathrm{CH}, \mathrm{PhCO}$

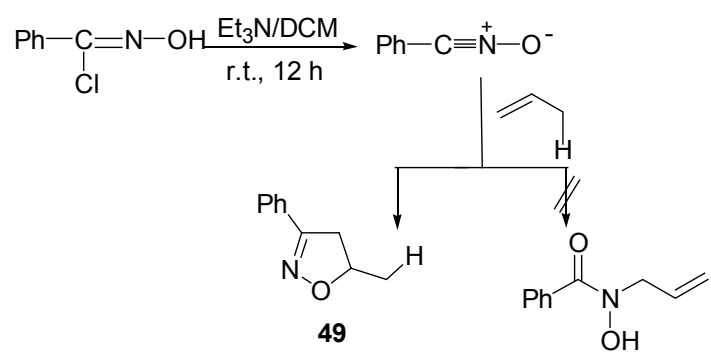

1,3-dipolar cycloadduct

Scheme 14

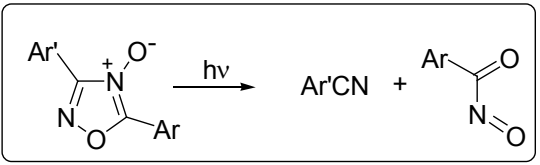<smiles>O/N=C(\Cl)c1ccccc1</smiles><smiles>CN1CCOCC1CO</smiles>

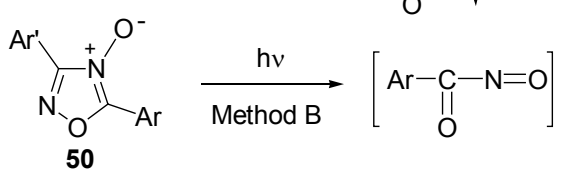<smiles>[R]C=C([R])C([R])([R])N(O)C(=O)[Al]C</smiles>

52

\begin{tabular}{cccc}
\hline Entry & Olefin & Yield (Method A) & Yield (Method B) \\
\hline 1 & & $99 \%$ & $99 \%$ \\
2 & & & \\
\hline
\end{tabular}

Scheme 15
由于其他试剂存在于反应体系中，往往会影响底物中敏 感官能团. 因此，需要发展一种更为简便直接的方法来 制备亚硝基中间体，以利于该反应的推广应用.

2.2.1.5 氧化酰基着胺化合物来制备酰基亚硝基中 间体

虽然在常规的氧化条件下，亚硝基一烯反应的产率 很低. 但 Adam 等 ${ }^{[42]}$ 发现二醋酸碘苯是一个温和的氧化 剂, 可以获得很好的结果. 之后通过两步常规的化学转 化便可得到烯丙基酰胺类化合物 54 (Scheme 16). 他们 在实验中发现这个方法只适用于富电子的底物烯, 而对 于缺电子烯基底物的反应效率很低.

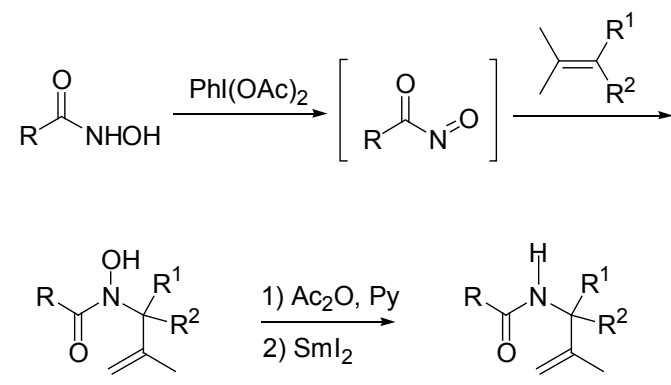

53

54

\begin{tabular}{cccc}
\hline Entry & Olefin & $\mathrm{R}$ & Yield/\% \\
\hline 1 & & $p-\mathrm{NO}_{2} \mathrm{C}_{6} \mathrm{H}_{4}$ & 82 \\
2 & MeOOC & $p-\mathrm{NO}_{2} \mathrm{C}_{6} \mathrm{H}_{4}$ & 10 \\
\hline
\end{tabular}

\section{Scheme 16}

2004 年 Iwasa 小组 ${ }^{[43]}$ 报道了新的酰基羟胺化合物 氧化的方法来制备酰基亚硝基中间体，即利用钌、铱或 铜催化剂在双氧水的条件下进行催化氧化(Eq. 1). 实验 中发现苯甲酰基着弪胺酸 55a 不适用于此条件, 而羟胺甲 酸叔丁酯 55b 则能顺利进行反应. 对于底物 2-甲基苯乙 烯 56b, 只有用碘化亚铜作为催化剂时才能发挥作用.

2011 年, Malkov 等 ${ }^{[44]}$ 在 Iwasa 工作的基础上, 发展 了一种更为温和和简便的方法, 用三氯化铁作催化剂, 双氧水作氧化剂，实现了分子内亚硝基一烯反应，他们 发现空间位阻对反应有很大影响，对于异丙基取代的烯 基底物即使升温至 $100{ }^{\circ} \mathrm{C}$ 也无法得到反应产物 59c. 当 差胺甲酸酯 $\alpha$ 位有取代基时，立体选择性不是很理想， 只有对于生成带有季碳手性中心的产物 $60 \mathrm{c}$ 时给出优异 的非对映选择性. 此外, 利用这种方法还可以构建螺环 化合物 59b (Scheme 17), 具有很好的应用价值.

同年 Read de Alaniz 小组 ${ }^{[45]}$ 也发表了用氯化亚铜作 为催化剂，空气作氧化剂的方法来制备酰基亚硝基中间 


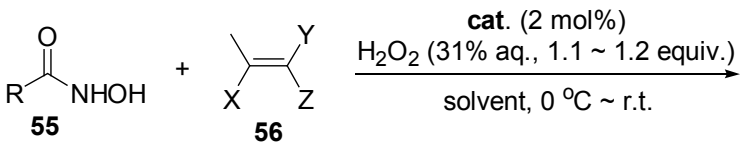<smiles>[Y]C(=C)C([X])(N(O)C([R])=O)C([Y])([Y])O</smiles>

55a: $\mathrm{R}=\mathrm{Ph}$, 55b: $\mathrm{R}=\mathrm{OBu}-t$

56a: $X=Z=\mathrm{CH}_{3}, Y=H$; 56b: $X=P h, Y=Z=H$;

56c: $X=Z=Y=\mathrm{CH}_{3}$

cat. $\left[\operatorname{lr}(\mathrm{coe})_{2} \mathrm{Cl}\right]_{2},[\operatorname{Ir}(\mathrm{cod}) \mathrm{Cl}]_{2}, \mathrm{Ru}($ pybox-dh)(pydic), Cul

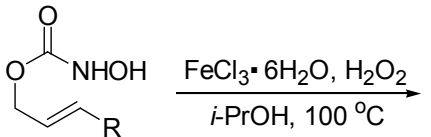<smiles>CC/C=C/C1COC(=O)N1O</smiles>

59a $64 \%$

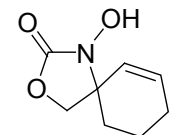

59b $65 \%$ or<smiles>CC(C)=CC1COC(=O)N1O</smiles>

59c $0 \%$<smiles>[R]C(/C=C\C)OC(=O)NO</smiles><smiles>C=CC1C(C)OC(=O)N1O</smiles>

60a $58 \%, d r=2: 1$

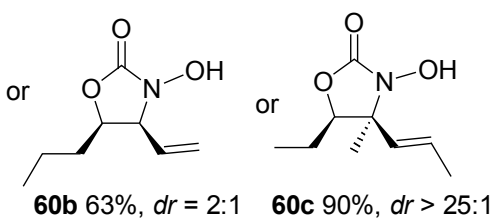

Scheme 17
体. 这种方法不仅可以促进分子内亚硝基 ene 反应，同 时对分子间亚硝基一烯反应也取得了优异的结果，同时 也能降低烯基底物的用量. 作者还对底物范围进行了探 索, 发现这个条件能容忍底物中多种官能团, 即使缺电 子底物或有氨基取代的底物都能获得很好的反应效果 (Scheme 18). 除此之外, 他们小组 ${ }^{[46]}$ 发展的这种制备酰 基亚硝基中间体的方法还可以应用于 Diels-Alder 反应, 同时亚硝基底物类型得到了进一步的拓展.

2012 年, 在这一领域最新的进展是 $\mathrm{Lu}$ 小组 ${ }^{[47]}$ 利用 双铑催化剂在等物质的量过氧叔丁醇的条件下来制备 酰基亚硝基中间体(Scheme 19). 在仅有 $0.1 \mathrm{~mol} \%$ 催化 剂的作用下于室温便能以最高 $96 \%$ 的产率得到杂 Diels-Alder 环加成的产物, 但他们并没有对 ene 反应进 行尝试. 考虑其温和的反应条件及较低催化剂用量, 可 以将这一方法拓展至亚硝基-烯反应领域.<smiles>[R]Cc1ccc([R])c(=O)o1</smiles>
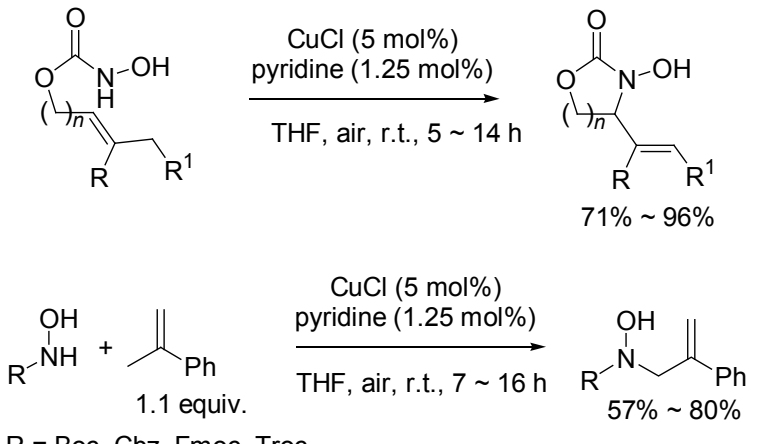

$\mathrm{R}=\mathrm{Boc}, \mathrm{Cbz}$, Fmoc, Troc

\section{Scheme 18}
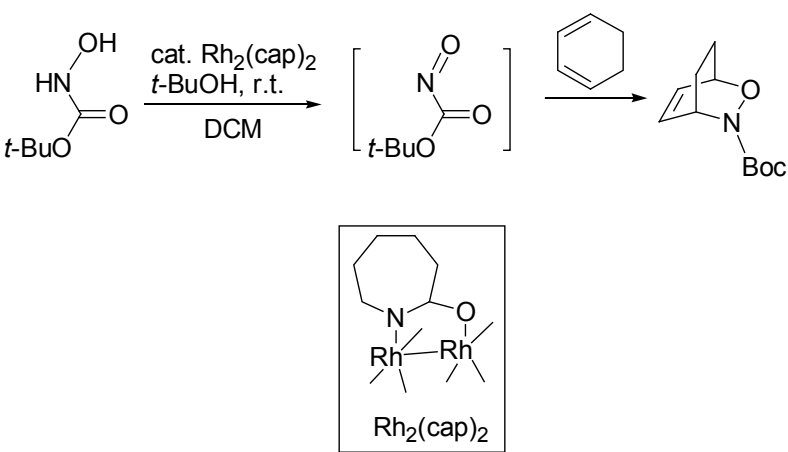

Scheme 19

\subsubsection{6 涉及亚硝基-烯反应的烯丙基胺化反应}

近年来, 人们对过渡金属催化的烯丙基胺化反应较 为关注 ${ }^{[18 b]}$. 对于涉及亚硝基中间体的反应，分为氧化 烯丙基胺化和还原烯丙基胺化反应两种. 在氧化烯丙基 胺化反应中, 羟胺苯被过渡金属氧化成亚硝基苯，之后 金属又会被烯反应产物羟胺化物氧化从而完成催化循 环. 在还原烯丙基胺化反应中，硝基苯被过渡金属还原 来进行亚硝基一烯反应，而金属又会被一氧化碳还原. 这两类反应生成的产物均是烯丙基胺，而不是一般亚硝 基一烯反应的烯丙基羟胺化合物.

在氧化烯丙基胺化反应中, 铁、钿和铜都是合适的 金属催化剂 ${ }^{[48]}$. 早在 1978 年, Sharpless 等 ${ }^{[49]}$ 就发现了钿 络合物催化的烯丙基胺化反应，但他们并未进行深入的 研究. 对这类反应的机理, Nicholas 等认为是过渡金属 的氧化还原催化循环(Scheme 20). 首先高价金属络合 物把羟胺底物 64 氧化成亚硝基苯(可以与金属中心配 位，也可以游离)，发生烯反应后生成的羟胺化合物作 为氧化剂将四价的钼氧化成六价, 从而完成催化循环. 反应最终生成的是烯丙基胺化合物. 虽然反应的机制现 在还不清楚，但是 Sharpless 等 ${ }^{[49]}$ 分离到钅与亚硝基苯 的络合物的单晶，也支持了这一可能的反应历程. 
Sharpless's reaction:

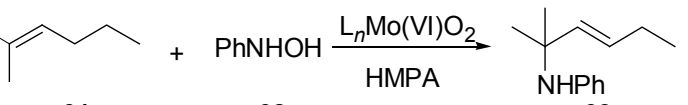

61

62

63

Proposed mechanism:

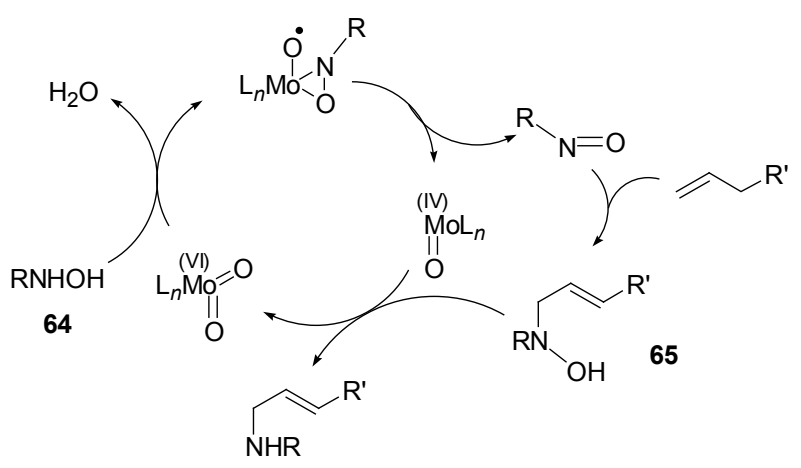

Scheme 20

对于还原烯丙基胺化反应，文献中报道的例子不 多, 对其机理和应用也没有过多的探索 ${ }^{[50]}$. 通常人们认 为金属催化剂在反应中对硝基底物 67 和烯反应产物均 是起还原作用，而一氧化碳则还原金属使其完成催化循 环, 但其反应机理至今仍没有文献报道. 虽然这是一种 简便的烯丙基胺化方法, 但需要较高的反应温度和较高 压力的 $\mathrm{CO}$ 气氛下进行, 而且底物范围一般受限于芳香 基取代的烯丙基底物(Eq. 2).

$$
\underbrace{\mathrm{R}}_{66}+\underset{67}{\mathrm{ArNO}_{2}}+\mathrm{CO} \stackrel{\text { Conditions }}{\longrightarrow} \mathrm{ArHN}_{68} \mathrm{R}
$$

Conditions:

$\mathrm{A}:\left[\mathrm{Ru}(\mathrm{CO})_{12}\right], 160{ }^{\circ} \mathrm{C}, 4.05 \mathrm{MPa} \mathrm{CO}$

B: $\left[\mathrm{CpFe}(\mathrm{CO})_{2}\right]_{2}, 160^{\circ} \mathrm{C}, 5.06 \mathrm{MPa} \mathrm{CO}$

C: $\left[\mathrm{Cp}{ }^{*} \mathrm{Fe}(\mathrm{CO})_{2}\right]_{2}, \mathrm{~h} v, 80 \sim 120^{\circ} \mathrm{C}, 0.202 \sim 0.606 \mathrm{MPa} \mathrm{CO}$

Beifuss 等 ${ }^{[51]}$ 在微波促进的条件下, 利用亚磷酸三 乙酯作为还原剂, 将芳基硝基底物还原生成亚硝基活性 中间体, 随后和底物中的双键发生烯反应, 实现了 benzoxazines, quinoxalines 和 quinolines 杂环化合物的 构建(Eq. 3). 之后作者又对反应条件进行了优化, 加入 钿金属催化剂并使用三苯基膦作为还原剂，使产率得到 进一步提高 ${ }^{[52]}$.

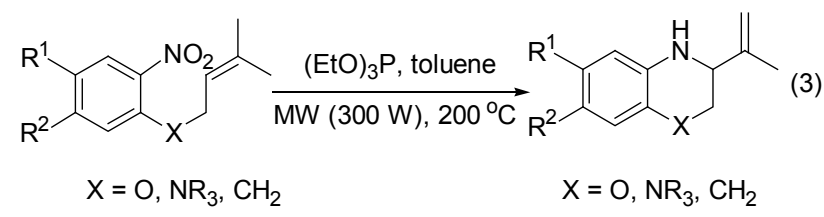

\subsection{2 亚硝基底物的发展}

正如之前提到的, 使用缺电子的亚硝基化合物作
为亲电试剂进行亚硝基一烯反应时，可以解决烯产物继 续发生原位反应的问题. 因此人们通常选择酰基亚硝 基、 $\alpha$ 卤代亚硝基以及缺电子的杂环亚硝基化合物用于 亚硝基-烯反应. 酰基亚硝基在上一节已经详细介绍, 其他类型的亚硝基底物表现出不同的反应活性.

\subsubsection{1 烷基亚硝基底物}

1972 年, Roberts 等 ${ }^{[53]}$ 报道了烷基亚硝基参与的烯 反应，也是第一例分子内亚硝基-烯反应. 利用偶氮二 甲酸乙酯或碳酸银氧化差胺化物 69 生成亚硝基中间体, 随后与烯烃发生分子内的亚硝基一烯反应得到环化产物 70 (Scheme 21)，但产率通常只有 $20 \% \sim 30 \%$.

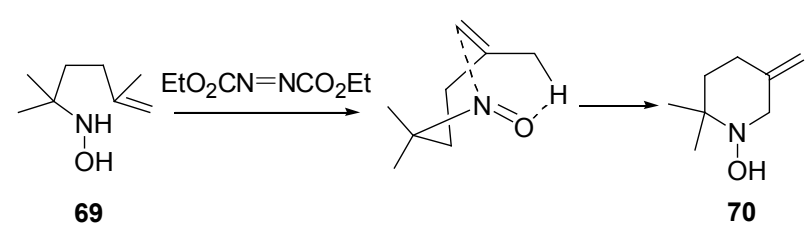

Scheme 21

另一例烷基亚硝基 ene 反应的发现则比较偶然, Solomon 等 ${ }^{[54]}$ 本想探索自由基聚合反应，却意外得到了 $23 \%$ 的亚硝基一烯反应产物. 他们推测是单电子氧化转 移机理, TEMPO 在苯甲酸根阴离子作用下发生碎裂化 反应得到含有分子内双键的亚硝基化合物 73 , 然后再 发生亚硝基一烯反应并继续氧化得到硝酮产物 $\mathbf{7 5}$ (Scheme 22).

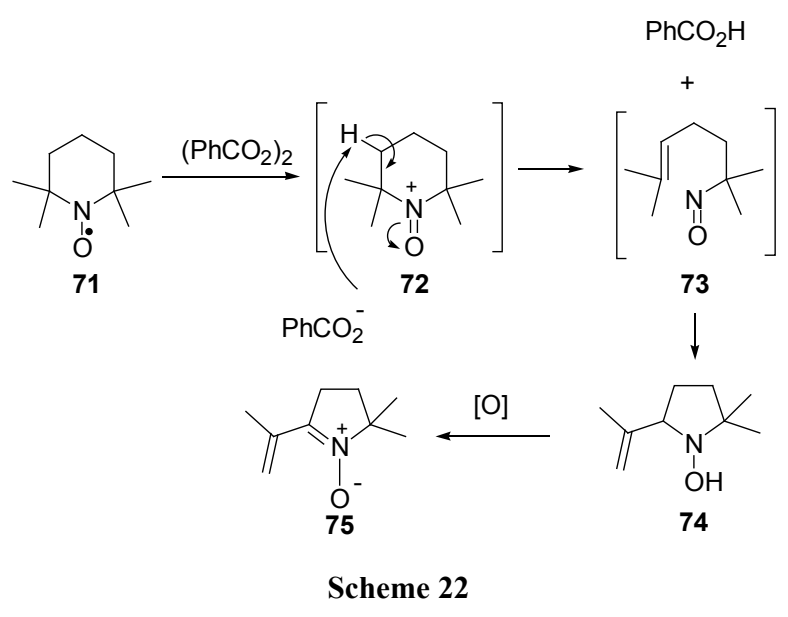

\subsubsection{2 $\alpha$ 卤代亚硝基底物}

随着亚硝基亲电性的增加，反应活性也会提高. 亚 硝基三氟甲烷便是活性最高的底物之一，不管较少取代 还是多取代的烯烃底物都能以很高的产率获得加成产 物. 对于亚硝基五氟苯同样也有很好的结果, 即使缺电 子烯烃也能以 $41 \%$ 的产率分离到加成产物 ${ }^{[55]}$ (Scheme 23). 

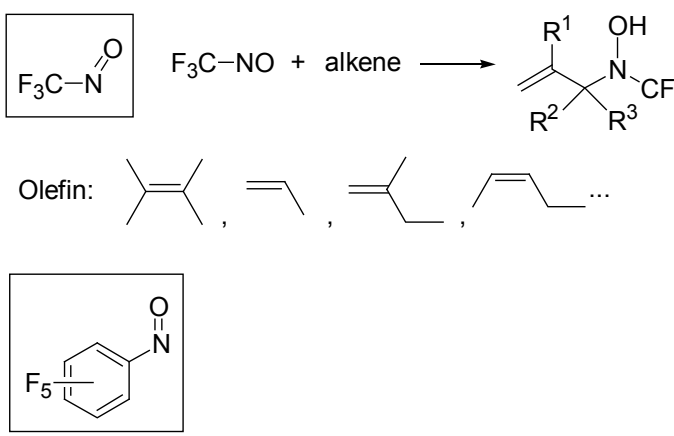

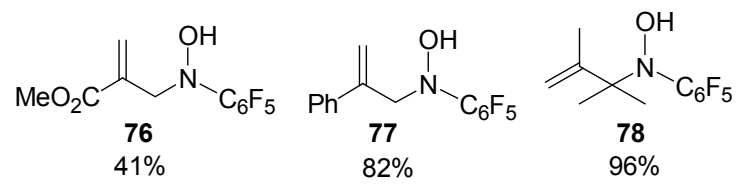

\section{Scheme 23}

1979 年, De Boer 和 Schenk ${ }^{[56]}$ 报道了 $\alpha$-氯代亚硝基 金刚烷与 $\alpha$-甲基苯乙烯的亚硝基一烯反应. 实验中他们 并未分离到烯反应产物 80 , 而是氯消除的产物硝酮 81 (Scheme 24). 幸运的是由于产物是盐酸盐, 会直接从体 系中析出, 从而防止了其进一步的被原位氧化. 之后他 们对亚硝基底物范围进行了探索, 尽管收率皆不错, 但 反应时间仍需 $12 \mathrm{~h}$ 以上, 源于这类亚硝基底物的反应活 性较低.

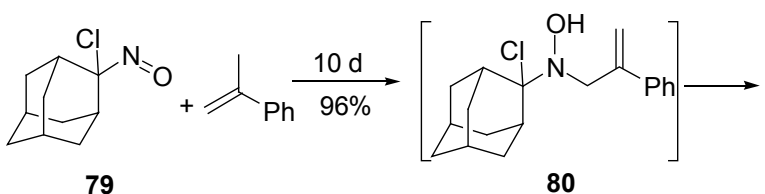

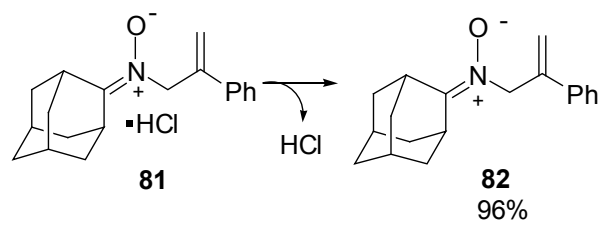

$$
\begin{array}{|ll}
\mathrm{R}^{2} \mathrm{Cl} & \begin{array}{l}
\mathrm{R}^{1}=\mathrm{R}^{2}=\mathrm{CH}_{3}, 91 \% \\
\mathrm{R}^{1}=\mathrm{CH}_{3}, \mathrm{R}^{2}=\mathrm{C}_{2} \mathrm{H}_{5}, 79 \%
\end{array} \\
\mathrm{R}^{1} \mathrm{~N}=\mathrm{O} & \begin{array}{l}
\mathrm{R}^{1}=\mathrm{CH}_{3}, \mathrm{R}^{2}=\mathrm{C}_{6} \mathrm{H}_{5} \mathrm{CH}_{2}, 73 \% \\
\mathrm{R}^{1}-\mathrm{C}-\mathrm{R}^{2}=c-\mathrm{C}_{6} \mathrm{H}_{10}, 76 \%
\end{array}
\end{array}
$$

\section{Scheme 24}

\subsubsection{3 吡啶及异噁唑类亚硝基底物}

2010 年, Miller 小组 ${ }^{[57]}$ 报道了用吡啶及异啞唑类亚 硝基作为底物的亚硝基一烯反应. 对各种底物可以以中 等到优秀的产率获得目标产物(Eq. 4).

\section{3 亚硝基-烯反应的应用}

1981 年, Keck 等 ${ }^{[58]}$ 将亚硝基一烯反应用于生物碱 crinane 的消旋体全合成(Scheme 25). 由直接购得的化

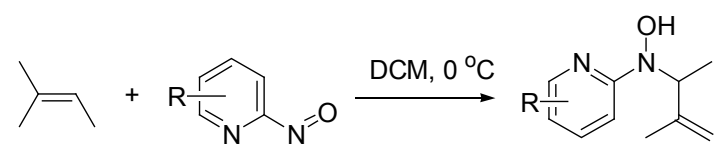

$38 \% \sim 94 \%$

合物 83 出发, 经 10 步得到着胺酸化合物 84, 用四正丙 基高碘酸铵将其氧化成亚硝基并被 9,10-二甲基蒽 (DMA) 捕获，之后在甲苯回流的条件下发生逆 Diels-Alder 反应释放出酰基亚硝基中间体 $\mathbf{8 5}$, 再与分子 内双键进行亚硝基一烯反应得到顺式的六并五环状结构 86. 经过三步化学转化得到仲胺 $\mathbf{8 8}$, 最后利用 Pictet-Spengler 反应生成消旋的 crinane. 合成策略中巧 妙利用立体专一性的亚硝基一烯反应所构建的六并五环 状化合物 86 是一类非常重要的合成中间体，它不仅是 同家族其他生物碱的合成中间体，也是多个具有生物活 性分子的核心单元 ${ }^{[59]}$. 对于同家族成员 mesembrine ${ }^{[60]}$ 和 dihydromaritidine ${ }^{[61]}$ 同样可以使用亚硝基-烯反应作 为关键反应来构建其核心骨架.

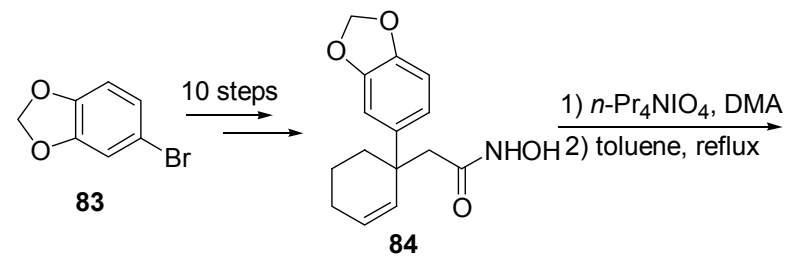

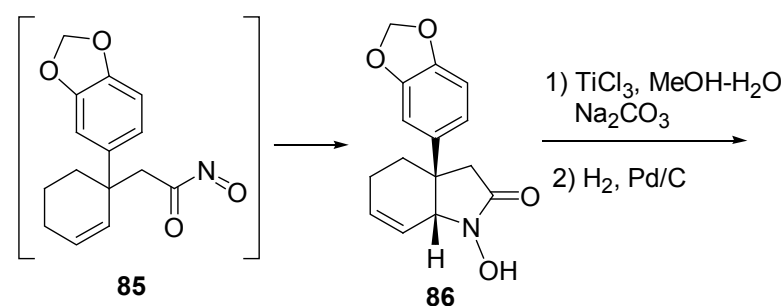

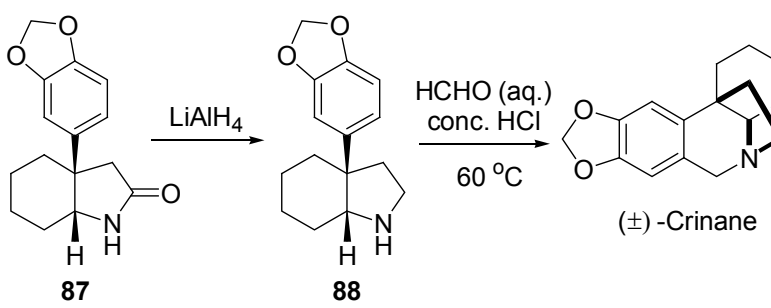

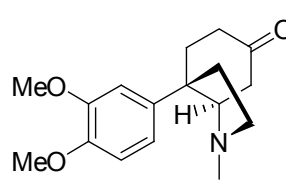

Mesembrine

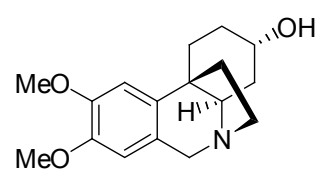

Dihydromaritibrine
Scheme 25

2003 年, Kibayashi 小组 ${ }^{[62]}$ 报道了利用分子内亚硝 基一烯反应高效构建海洋生物碱 halichlorine 及结构类似 的 pinnaic acid 的核心螺环骨架(Scheme 26). 从 2-环戊 烯䣶出发, 经过五步得到差胺酸化合物 89 , 在冰水浴中 
用四正丙基高碘酸铵将其氧化成亚硝基中间体并与分 子内的双键进行烯反应, 由于烯丙位的着基手性控制, 亚硝基以 $S i$-面实现烯反应, 得到 halichlorine 及 pinnaic acid 中的正确构型的核心骨架螺环化合物 90. 通过五元 环上手性羟基的引入可以诱导不对称亚硝基-烯反

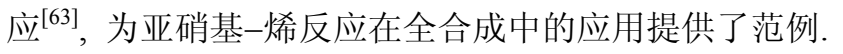

2007 年, Vasella 等 ${ }^{[64]}$ 报道了利用亚硝基-烯反应作 为关键步骤来合成碟啶类化合物的方法(Scheme 27). 从亚硝基嘧啶类化合物 91 出发, 根据目标产物需要引
入不同酰胺取代基，之后进行亚硝基-烯反应再脱水得 到碟啶类化合物. 应用烯反应的策略, 可以方便地合成 类似结构的药物分子如 ciliapterin, umanopterin, dictyopterin 和 biopterin ${ }^{[65]}$. 大大拓展了亚硝基一烯反应在 复杂底物中的应用和对敏感官能团的容忍性.

对于抗疮药 FR900098 (94)的合成, 若引入亚硝基一 烯反应，则仅需 5 步便可以 $64 \%$ 的总产率获得 ${ }^{[66]}$ (Scheme 28). 其中乙酰基亚硝基中间体是通过逆 DielsAlder 得到的.
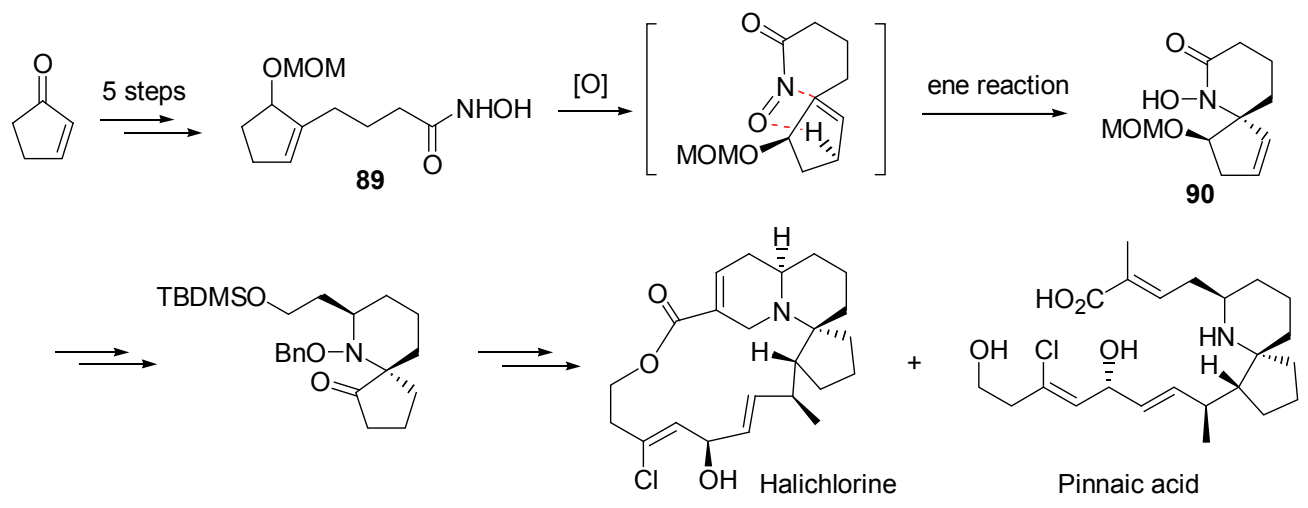

Scheme 26

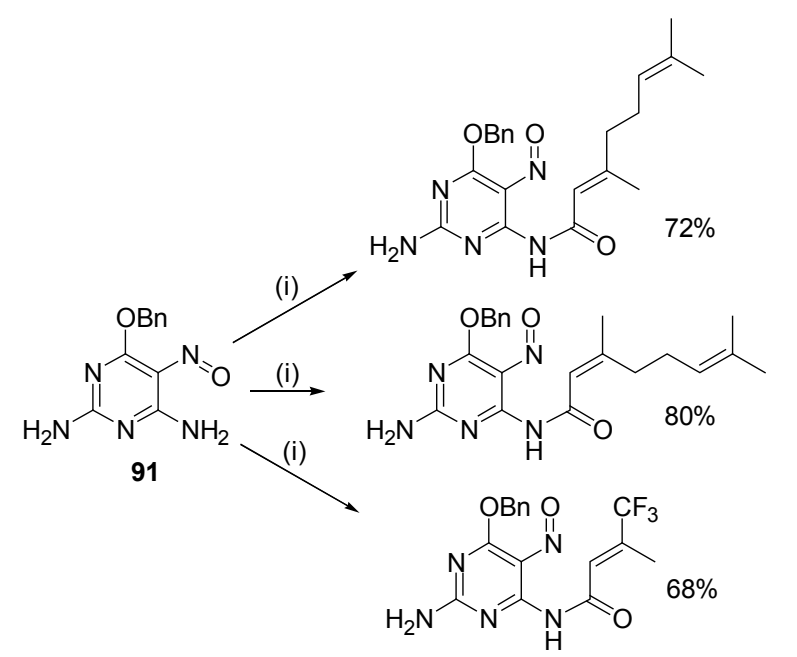<smiles>C=C(CCC=C(C)C)c1nc2c(OCc3ccccc3)nc(N)nc2[nH]c1=O</smiles>

Reagents and conditons: (i) 1.3 equiv. $\mathrm{RCOCl}, \mathrm{K}_{2} \mathrm{CO}_{3},-18{ }^{\circ} \mathrm{C}$, THF; (ii) sol. in $\mathrm{CH}_{2} \mathrm{Cl}_{2}, 4 \mathrm{~d}$; (iii) suspension in toluene, $150{ }^{\circ} \mathrm{C}, 8 \mathrm{~h}$ in sealed vial

\section{Scheme 27}
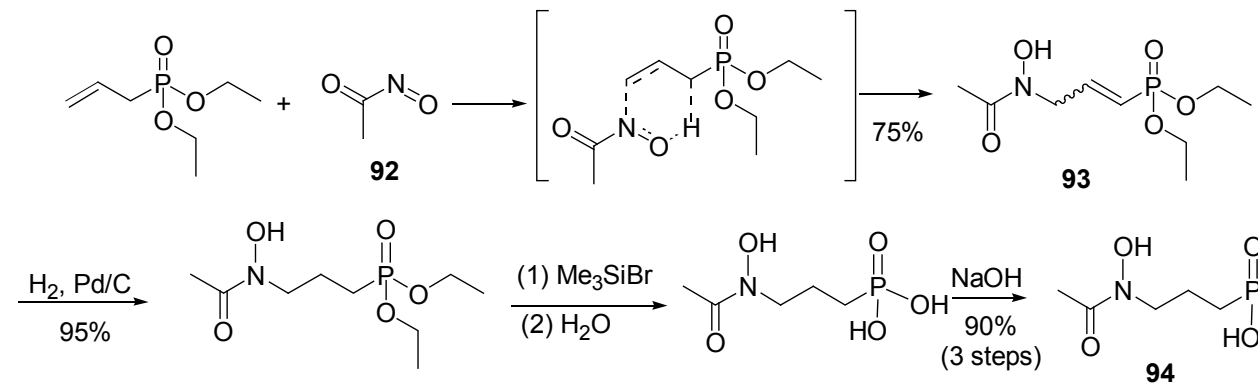<smiles>CC(=O)N(O)CCCP(=O)(O)O</smiles>

\section{Scheme 28}


2008 年, Haley 小组 ${ }^{[67]}$ 报道了利用串联的亚硝基-烯 及分子内环化反应来构建苯并异噁唑亚胺化合物 97 (Scheme 29). 首先利用臭氧化得到亚硝基化合物 96, 再 与四甲基乙烯进行烯反应和随后的分子内环化得到产 物 97. 此外, 他们也发现产物苯并异噁唑亚胺化合物 97 在 $\mathrm{HBF}_{4}$ 作用下会发生二聚得到新化合物 98.

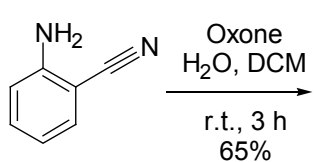

95

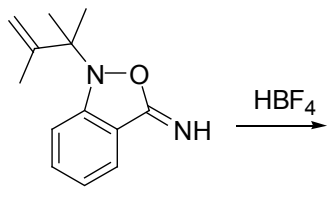

97

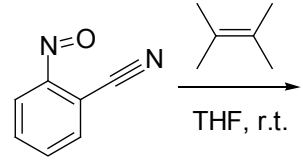

96

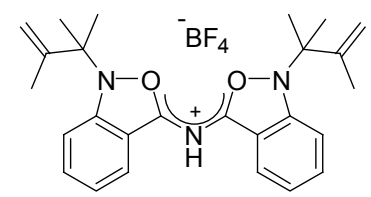

98

\section{Scheme 29}

2012 年, Srivastava 小组 ${ }^{[68]}$ 采用涉及亚硝基-烯反应 的烯丙基胺化反应来合成氮杂 Baylis-Hillman 产物. 羟 胺苯先被二氯化铁氧化成亚硝基苯随后与 $\alpha, \beta$-不饱和羰 基化合物进行烯反应，之后羟胺化产物又会被低价铁继 续还原成二级胺也就是氮杂 Baylis-Hillman 产物, 同时 再生催化剂, 完成催化循环(Eq. 5). 在亚硝基-烯反应这 一领域中, 对于缺电子的烯基底物往往难以获得优异的 结果. 而在这一工作中, 尽管均是酮或酯等缺电子底物, 但都能得到较好产率的加成产物. 这对亚硝基-烯反应 的底物类型是很好的拓展.

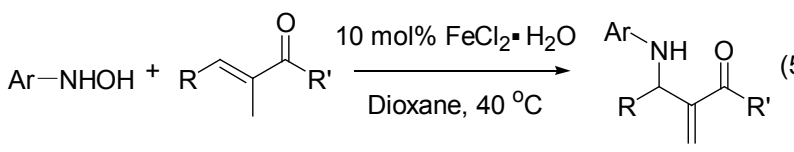

\section{3 亚硝基-烯反应的机理研究}

\section{1 亚硝基-烯反应的机理}

对反应机理的深入研究, 不仅为我们理解反应的 速控步，过渡态的结构和取代基对反应的影响，也对有 助于发展不对称催化的亚硝基-烯反应和拓展其应用. 尽管文献中对于亚硝基参与的杂 Diels-Alder 反应一般 认为是协同反应 ${ }^{[35]}$, 但对于亚硝基-烯反应的机理却一 直存在争论. 至今为止, 文献中已经提出了有 6 种不同 的反应机理(Scheme 30), 其中包括一种环状过渡态的 协同机理以及 5 种分步机理. 除了最后经过高张力四元 环和双自由基机理的途径 e, f 可能性较小外 ${ }^{[69]}$, 其他 4 种皆是亚硝基-烯反应可能的机理均有一定实验数据或
理论的支持.

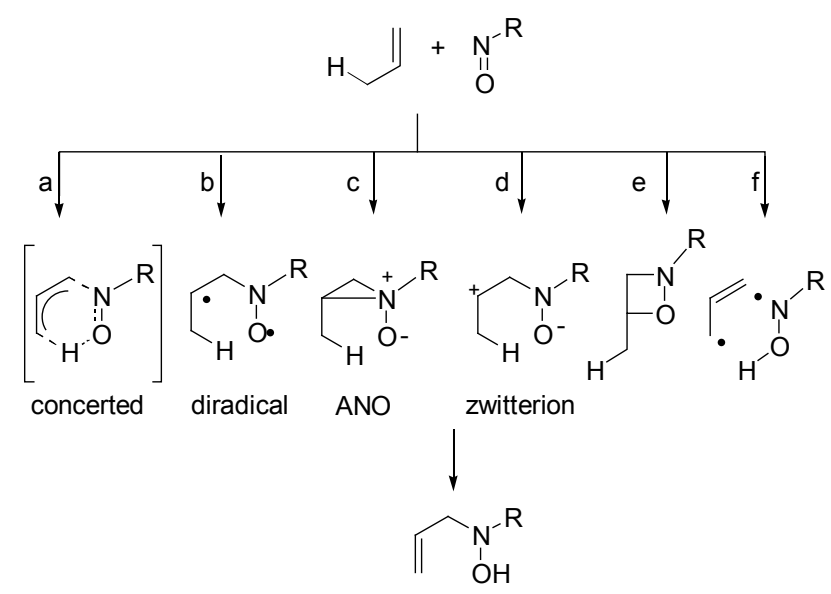

Scheme 30

1982 年, Greene 小组 ${ }^{[70]}$ 首次报道了对亚硝基-烯反 应的机理研究. 他们用亚硝基五氟苯作为亲电试剂, 氛 代四甲基乙烯作为烯烃底物，根据同位素效应的结果来 推测烯反应的机理(Scheme 31). 在实验中发现, 顺式的 $d_{6}$-四甲基乙烯没有同位素效应 $\left(k_{\mathrm{H}} / k_{\mathrm{D}} \approx 1\right)$, 而反式 $d_{6}$-四 甲基乙烯则有 $\left(k_{\mathrm{H}} / k_{\mathrm{D}} \gg 1\right)$. 因此作者认为亚硝基-烯反 应的第一步生成氧代氮杂环丙烷(ANO)三元环中间体 是不可逆的，也是整个反应的决速步，然后再通过㩲氢 得到烯反应产物. 之后 Schiesser 等 ${ }^{[71]}$ 根据 PM3 理论计 算认为丙烯与亚硝酰的烯反应的中间体氧代氮杂环丙 烷是可能的中间体，为这一反应机理提供了理论支持.

2003 年, Krebs 等 ${ }^{[72]}$ 同样用気代实验对亚硝基-烯反 应进行了更为细致深入的研究. 他们利用分子间同位素 实验对比作为参照，发现反式及同碳 $d_{6}$-四甲基乙烯的 同位素效应 $\left(k_{\mathrm{H}} / k_{\mathrm{D}}=3 \sim 4\right)$ 要大于分子间同位素动力学常 数比 $\left(k_{\mathrm{H}} / k_{\mathrm{D}}=2\right)$. 由此可以推断, 氧代氮杂环丙烷 (aziridine $N$-oxide, ANO)的形成是可逆的. 此外他们还 进行了深入的动力学研究, 并计算出可逆动力学常数与 篗氢及箱氝动力学常数的关系 $k_{\mathrm{r}}=k_{\mathrm{a}}(\mathrm{D})=1 / 3 k_{\mathrm{a}}(\mathrm{H})$ (Scheme 32).

Houk 等 ${ }^{[73]}$ 最近的理论计算表明，虽然氧代氮杂环 丙烷的过渡态模型是可能的过程，但是极化双自由基 (PD)的模型(Scheme 33)具有更低的能量状态, 也能解释 之前同位素效应的实验数据. 同时, 更具经验的 B2LYP/6-31g*计算方法也支持了丙烯与亚硝酰的烯反 应中极化双自由基(PD)中间体的形成 ${ }^{[74]}$. PD 模型和 ANO 模型过渡态的能量差异很小，意味着反应经过两 种机理均有可能. 同时 DFT 计算无法锁定 WoodwardHoffmann 规则中的协同反应的过渡态能量，因此可以 认为协同反应机理是很不利的. 在他们的计算中同时也 指出，虽然双自由基一般认为是很难给出好的立体选择 


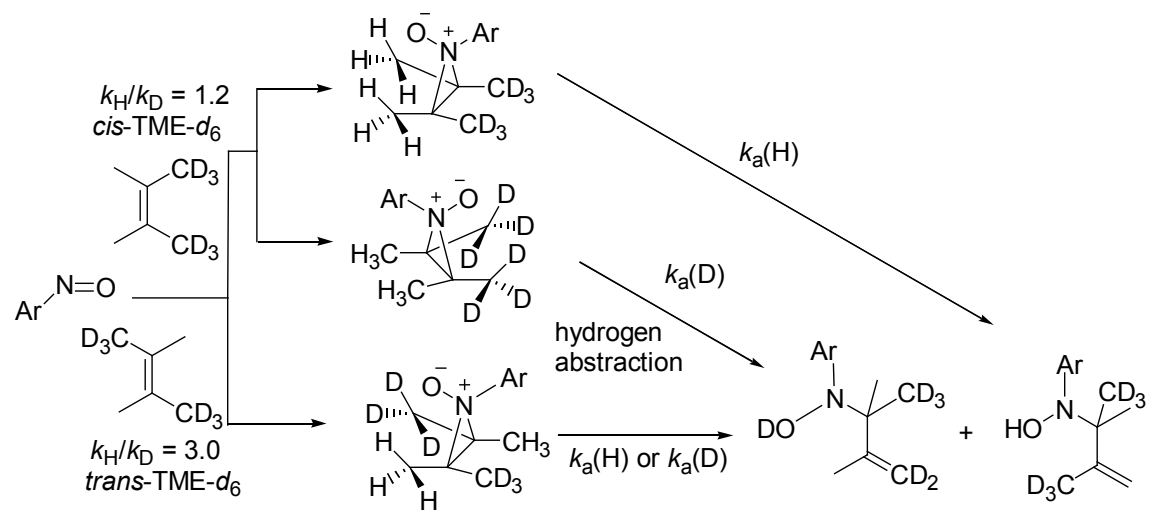

Scheme 31

Enophile Intermolecular

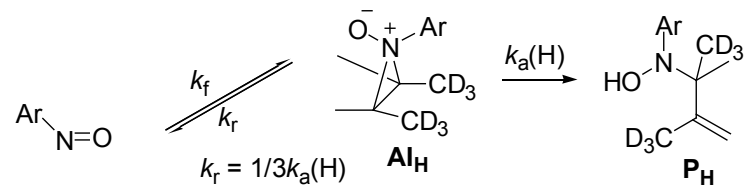<smiles>CCCCCCCC(C)=C(C)C(C)(C)C</smiles>

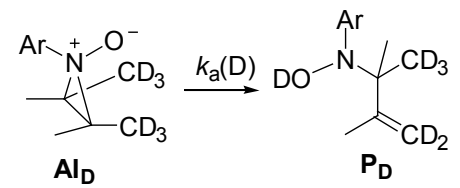

\section{Scheme 32}

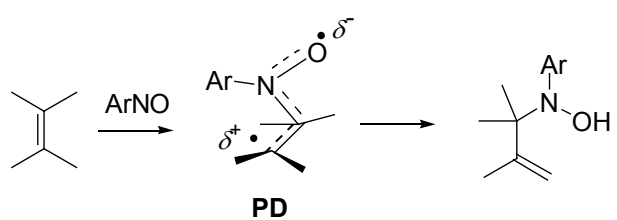

Scheme 33

性，但是这里的亚硝基-烯反应过程中产生的双自由基 是极化的, 具有部分两性离子的性质 (zwitterioinic $\left.{ }^{-} \mathrm{O}-\mathrm{N}-\mathrm{C}-\mathrm{C}^{+}\right)$和弱的氢键作用. 这使得单键旋转的能垒稍 高于双自由基的形式, 也就是说能垒稍高于后续算氢的 步骤! 从这里可以看出, 双自由基的反应机理要比我们 通常想象的要复杂的多.

尽管大多数人认为亚硝基-烯反应是分步进行的, 但 $\mathrm{Lu}$ 等 ${ }^{[75]}$ 针对特殊烯烃底物 99 和亚硝基甲烷或对硝基 亚硝基苯的烯反应，提出协同的反应机理模型. 他们利
用密度泛函理论计算出协同的环状过渡态时其活化自 由能最低，仅 $67.4 \mathrm{~kJ} \cdot \mathrm{mol}^{-1}$, 因此推测协同反应为最优 路径(Scheme 34).

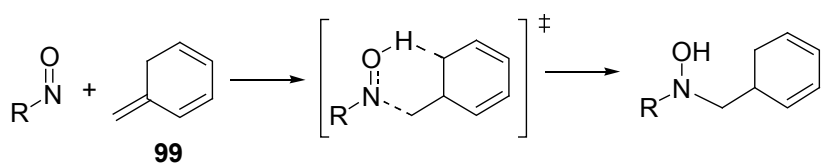

Scheme 34

\section{2 亚硝基一烯反应的区域选择性}

尽管反应机理还没有定论，但对于实验结果的分析 来看, 氧代环乙亚胺(ANO)三元环的过渡态模型能较好 地解释亚硝基一烯反应的区域选择性，也为我们对该反 应的设计和应用提供一定的理论依据. 
Adam 等 ${ }^{[76]}$ 为了更好地说明反应的区域选择性, 定 义空间位阻最大的一端为 twix 位, 同碳上空间位阻较小 的为 twin 位, 而对位的另外一端则是 lone 位(Scheme 35). 他们用对硝基亚硝基苯与各种底物烯反应, 发现 除了 107 之外, 其他底物均是以篗取 twix 位的氢为主要 产物. 这种倾斜效应可以从反应的过渡态中得到解释, 由于硝基苯取代基与底物双键上取代基的空间位阻排 蚱作用, 使氧更倾向 twix 位并最后完成篗氢过程而得到 烯反应产物.

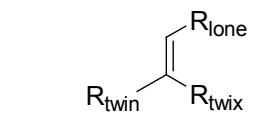

$5 \% \sim 17 \% \quad 95 \% \sim 83 \%$

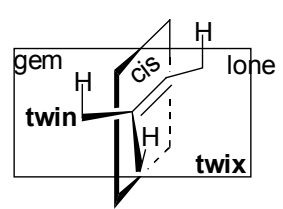

$\mathrm{R}_{\text {lone }}=\mathrm{Me}$, Et, $i-\mathrm{Pr}, t-\mathrm{Bu}, \mathrm{Bn}, \mathrm{Ph}, p-\mathrm{MeOC}_{6} \mathrm{H}_{4}, p-\mathrm{MeC}_{6} \mathrm{H}_{4}, p-\mathrm{MeC}_{6} \mathrm{H}_{4}$
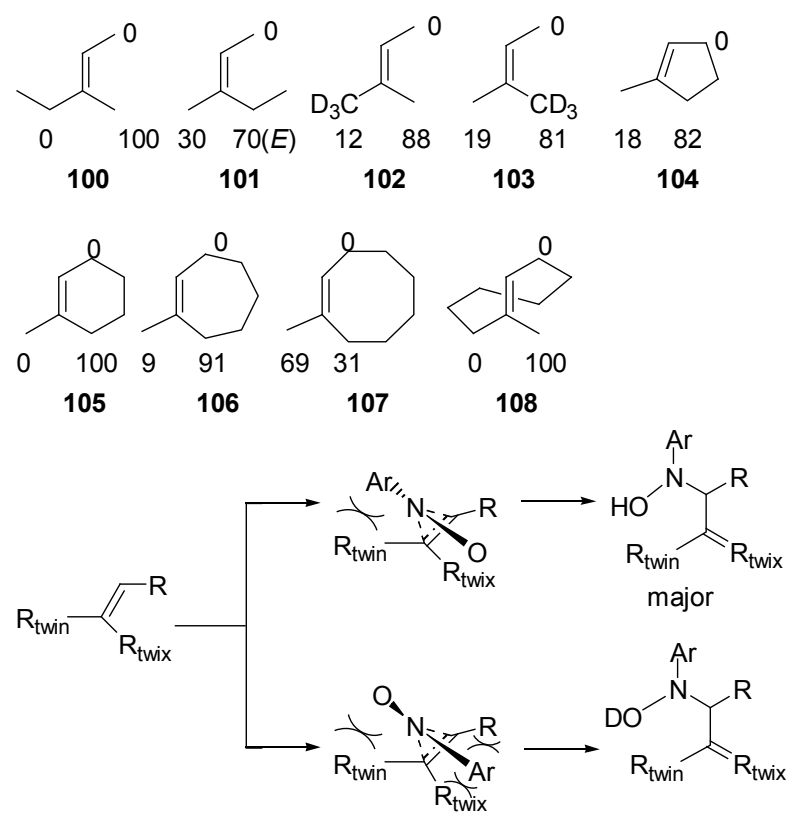

Scheme 35

之后，作者还尝试在含有两个双键的底物烯引入羟 基通过氢键作用来改变亚硝基 ene 反应的区域选择性 ${ }^{[77]}$ (Scheme 36). 结果发现, 含有羟基底物 2,3 位加成的产 物 111 确实有增加, 但从近 $1: 3$ 的比例中可以看出, 空 间位阻效应仍然是控制亚硝基一烯反应区域选择性最为 关键的因素.

\section{3 亚硝基-烯反应的立体选择性}

2000 年, Adam 等 ${ }^{[78]}$ 报道了利用底物羟基的氢键作 用来进行不对称亚硝基-烯反应(Scheme 37). 通过在双 键的烯丙位引入手性羟基来诱导烯烃底物与对硝基亚 硝基苯的不对称亚硝基一烯反应，得到高非对映选择性 $(>95: 5)$ 的加成产物 114.
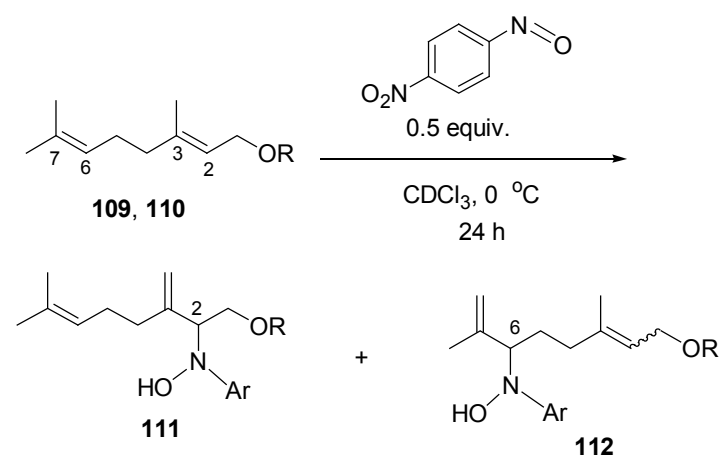

\begin{tabular}{ccc}
\hline Olefin & $\mathrm{R}$ & $\begin{array}{c}\text { Regioselectivity } \\
112: 111\end{array}$ \\
\hline 109 & $\mathrm{Me}$ & $95: 5$ \\
110 & $\mathrm{H}$ & $77: 23$ \\
\hline
\end{tabular}

Scheme 36

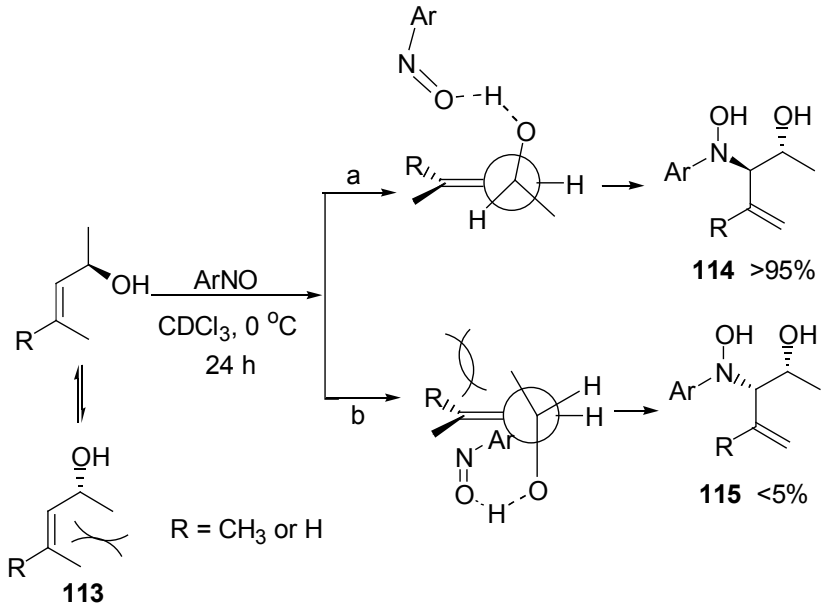

Scheme 37

1986 年, Vasella 小组 ${ }^{[79]}$ 报道了用带有手性糖的 $\alpha$ 氯 代亚硝基化合物尝试不对称亚硝基-烯反应(Scheme 38). 由亚硝基底物上的手性糖基来诱导进行亚硝基-烯 反应得到高非对映选择性的硝酮盐酸盐化合物 117 , 之 后经过酸处理和锂铝氢还原得到烯丙基胺化合物 119 , 同时产物的 $e e$ 值得到了保持.

2002 年, Adam 小组 ${ }^{[80]}$ 报道了利用带有手性辅基的 烯烃底物 120 与亚硝基化合物进行不对称亚硝基-烯反 应(Scheme 39). 他们在烯烃底物引入手性樟脑磺酰胺 类辅基，通过底物的手性诱导不对称的亚硝基一烯反应， 非对映选择性高于 $95 ： 5$. 之后通过两步简单的化学转 化得到手性 $\beta$-氨基酸，同时产物的 $e e$ 值可以大于 $95 \%$. 这是一种经济、高效且对映选择性高的合成 $\beta$-氨基酸的 方法.

2011 年 Read de Alanis 小组 ${ }^{[45]}$ 用氯化亚铜作为催化 剂，空气作氧化剂的方法来制备酰基亚硝基中间体进行 烯反应. 当在烯烃底物中同样引入手性樟脑磺酰胺类辅 
116a, 116b $\frac{\overbrace{\left(\mathrm{CH}_{2}\right)_{n}}}{20^{\circ} \mathrm{C}, 2 \sim 7 \mathrm{~d}}$

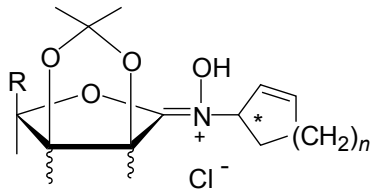

117

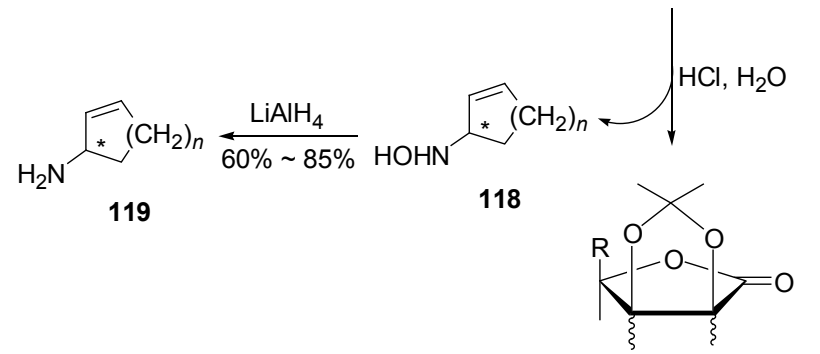

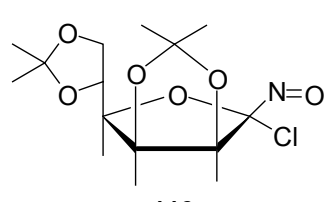

$116 a$
$\mathrm{Ph}_{3} \mathrm{CO}$

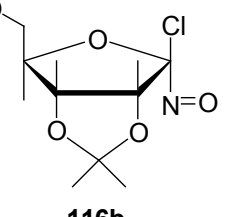

116b

\begin{tabular}{|c|c|c|c|c|c|}
\hline & $\mathrm{R}$ & $n$ & Yield $/ \%$ & $e e / \%$ & Conf. \\
\hline \multirow{2}{*}{$116 a$} & & 1 & 76 & 94 & $S$ \\
\hline & & 2 & 96 & 82 & $S$ \\
\hline \multirow{2}{*}{$116 b$} & $\mathrm{Ph}_{3} \mathrm{CO}$ & 1 & 70 & 96 & $R$ \\
\hline & & 2 & 91 & 96 & $R$ \\
\hline
\end{tabular}

\section{Scheme 38}
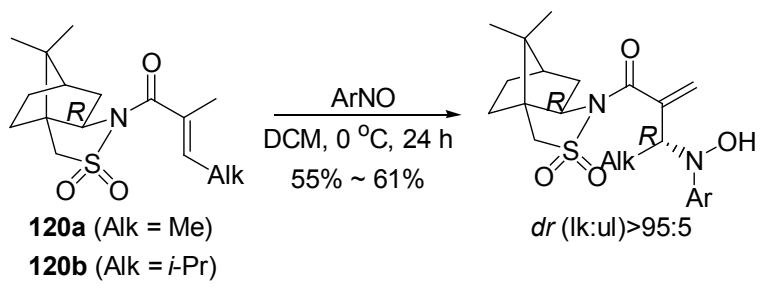

120b (Alk $=i-\mathrm{Pr})$

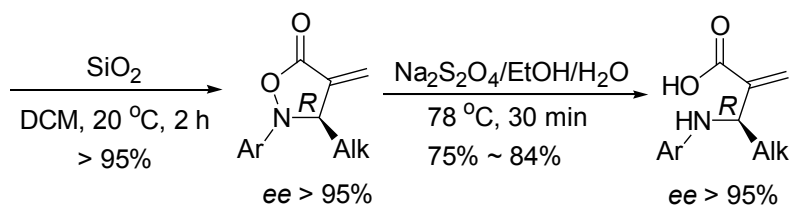

121a $($ Alk $=\mathrm{Me})$

121b $($ Alk $=i-\operatorname{Pr})$

\section{Scheme 39}

基, 便可以通过底物的手性诱导不对称的亚硝基-烯反 应，脱去手性辅基也得到高对映选择性(98.5：1.5)的化 合物 124 (Eq. 6).

以上的方法均是利用手性亲电试剂或手性烯烃底 物来诱导不对称亚硝基-烯反应. 2010 年, Miller 小组 ${ }^{[57]}$ 首次报道了催化不对称的亚硝基-烯反应(Eq. 7). 他们 利用一价铜和手性双磷配体作为催化剂，可以获得 $40 \%$

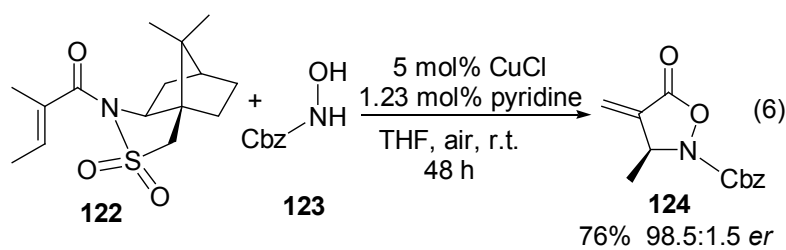

$e e$ 值. 虽然对映选择性不理想, 但是他们开创性的工作 会启发其他的研究小组设计出更优良的不对称催化体 系，真正将亚硝基一烯反应发展成为高效实用的有机合 成方法学.

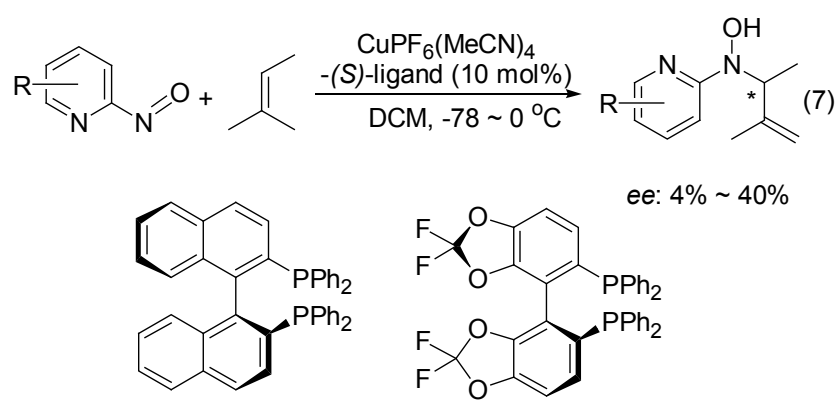

(S)-BINAP

(S)-DifluoroPhos

\section{4 总结和展望}

烯丙基胺类化合物是有机化学中的关键中间体，它 不仅是天然产物及药物中常见的结构单元，同时还能转 化成多种其他官能团. 而亚硝基一烯反应就是构建这类 烯丙基胺类化合物高效的方法之一. 虽然亚硝基-烯反 应早在 1965 年便被发现, 但由于潜在的应用价值直至 最近才得到化学们的广泛关注.

经过一些研究组的努力, 人们对亚硝基一烯反应有 了一定的认识，并在这一领域取得了一定的成果且成功 地将其应用于若干复杂化合物的合成中．但是如今仍有 很多问题没有解决，比如对于缺电子的烯基底物或是含 有多种官能团的底物还是不能得到很好的结果, 以及还 没有发展出高效实用的催化不对称的亚硝基一烯反应等. 这些问题亟待化学家们的持续努力和探索.

\section{References}

[1] (a) Meyer, V.; Locher, J. Ber. 1874, 7, 670.

(b) Meyer, V.; Locher, J. Ber. 1874, 7, 1506.

(c) Baeyer, A.; Caro, H. Ber. 1874, 7, 809.

(d) Baeyer, A. Ber. 1874, 7, 1638.

(e) Baeyer, A.; Caro, H. Ber. 1874, 7, 963.

[2] Gowenlock, B. G.; Richter-Addo, G. B. J. Chem. Educ. 2008, 85, 1243

[3] Lee, M. D.; Dunne, T. S.; Siegel, M. M.; Chang, D. D.; Morton, G. O.; Borders, D. B. J. Am. Chem. Soc. 1987, 109, 3464.

[4] Kersten, R. D.; Dorrestein, P. C. Nat. Chem. Biol. 2010, 6, 636

[5] Boyer, J. H. In The Chemistry of the Nitro and Nitroso Groups, Part 
1, Ed.: Patai, S., Interscience Publishers, New York, 1969, Chapter 5.

[6] Zuman, P.; Shah, B. Chem. Rev. 1994, 94, 1621.

[7] (a) Wichterle, O. Collect. Czech. Chem. Commun. 1947, 12, 292. (b) Arbuzov, Y. A. Dokl. Akad. Nauk SSSR 1948, 60, 993.

[8] Lewis, J. W.; Myers, P. L.; Ormerod, J. A. J. Chem. Soc., Perkin Trans. 1 1972, 2521.

[9] Banks, R. E.; Barlow M. G.; Haszeldine, R. N. J. Chem. Soc. 1965, 4714.

[10] For reviews, see: (a) Ager, D. J.; Prakash, I.; Schaad, D. R. Chem. Rev. 1996, 96, 835.

(b) Senanayake, C. H. Aldrichim. Acta 1998, 31, 3.

(c) Lait, S. M.; Rankic, D. A.; Keay, B. A. Chem. Rev. 2007, 107, 767.

[11] (a) Oppolzer, W.; Tamura, O. Tetrahedron Lett. 1990, 31, 991.

(b) Oppolzer, W.; Tamura, O.; Sundarababu, G.; Signer, M. J. Am. Chem. Soc. 1992, 114, 5900.

(c) Momiyama, N.; Yamamoto, H. Org. Lett. 2002, 4, 3579.

[12] Goelitz, P.; Meijere, A. Angew. Chem. 1977, 89, 892.

[13] (a) Aston, A.; Menard, M. J. Am. Chem. Soc. 1935, 57, 1922.

(b) Forrester, A. R.; Hepburn, S. P. J. Chem. Soc. C 1971, 3322.

(c) Goldman, J. Tetrahedron 1973, 29, 3833.

[14] Druellinger, M. L. J. Heterocycl. Chem. 1976, 13, 1001.

[15] (a) Adam, W.; Bottle, S. E.; Peters, K. Tetrahedron Lett. 1991, 32, 4283 .

(b) Torssell, K. Tetrahedron 1970, 26, 2759.

(c) Forrester, A. R.; Henderson, J.; Reid, K. Tetrahedron Lett. 1983, 24, 5547.

[16] (a) Ginsburg, V. A. J. Org. Chem. USSR (Engl. Trans.) 1974, 10, 1427.

(b) Barr, A.; Hazeldine, R. N. J. Chem. Soc. 1955, 1881.

[17] (a) Lin, C.-T.; Hsu, W.-J. Can. J. Chem. 1989, 67, 2153.

(b) Viehe, H. G.; Merenyi, R.; Francotte, E.; Van Meerssche, M.; Germain, G.; Declercq, J. P.; Bodart-Gilmont, J. J. Am. Chem. Soc. 1977, 99, 2340 .

(c) Gouverneur, V.; Dive, G.; Ghosez, L. Tetrahedron: Asymmetry 1991, 12, 1173.

(d) Christie, C. C.; Kirby, G. W.; McGuian, H.; Mackinnon, J. W. M. J. Chem. Soc., Perkin. Trans. 1 1985, 1972.

[18] (a) Knight, G. T. J. Chem. Soc., Chem. Commun. 1970, 1016. (b) Johannsen, M.; Jùrgensen, K. A. Chem. Rev. 1998, 98, 1689.

[19] (a) Glaser, R.; Murmann, R. K.; Barnes, C. L. J. Org. Chem. 1996, 61, 1047.

b) Stowell, J. C. J. Org. Chem. 1971, 36, 3055.

(c) Yamamoto, H.; Momiyama, N. J. Chem. Soc., Chem. Commun. 2005, 3514 .

[20] (a) Miller, M. J.; Bodnar, B. S. Angew. Chem., Int. Ed. 2011, 50, 5630 .

(b) Janey, J. M. Angew. Chem., Int. Ed. 2005, 44, 4292.

(c) Merino, P.; Tejero, T. Angew. Chem., Int. Ed. 2004, 43, 2995.

(d) Studer, A. Synthesis 1996, 793

(e) Voget, P. F.; Miller, M. J. Tetrahedron 1998, 54, 1323.

[21] Weinreb, S. M.; Staib, R. R. Tetrahedron 1982, 38, 3113.

[22] (a) Yamamoto, H.; Kawasaki, M. Bull. Chem. Soc. Jpn. 2007, 80, 595.

(b) Oppolzer, W.; Tamura, O.; Deerberg, J. Helv. Chim. Acta 1992, $75,1965$.

[23] Guo, H.; Cheng, L.; Cun, L. F.; Gong, L. Z.; Miac, A. G.; Jiang, Y. Z. J. Chem. Soc., Chem. Commun. 2006, 429.

[24] (a) Brown, S. P.; Brochu, M. P.; Sinz C. J.; MacMillan, D. W. C. J. Am. Chem. Soc. 2003, 125, 10808.

(b) Yanagisawa, A.; Arai, T. Chem. Commun. 2008, 1165.
[25] Mikami, K.; Shimizsu, M. Chem. Rev. 1992, 92, 1021.

[26] Alder, K.; Pascher, F.; Schimitz, A. Dtsch. Chem. Ges. 1943, 76, 27.

[27] (a) Magnus, P.; Lacour, J.; Goldham, I.; Mugrage, B.; Bauta, W. B. Tetrahedron 1995, 51, 11087.

(b) Seebach, D.; Overhand, M.; Kuèhnle, F. N. M.; Martinoni, B.; Oberer, L.; Hommel, U.; Widmer, H. Helv. Chim. Acta 1996, 79, 913.

(c) Cole, D. C. Tetrahedron 1994, 50, 9517.

(d) Abele, S.; Seebach, D. Eur. J. Org. Chem. 2000, 1.

(e) Burgess, K.; Liu, L.; Pal, B. J. Org. Chem. 1993, 58, 4758.

(f) Trost, B. M.; Van Vranken, D. L. J. Am. Chem. Soc. 1993, 115, 444.

[28] Banks, R. E.; Barlow, M. G.; Haszeldine, R. N. J. Chem. Soc. 1965, 4714.

[29] Keussler, V.; Luttke, W. Z. Elektrochem. 1959, 63, 614

[30] (a) Corrie, J. E. T.; Kirby, G. W.; Mackinnon, J. W. M. J. Chem. Soc., Perkin Trans. 1 1985, 883.

(b) Quadrelli, P.; Mella, M.; Invernizzi, A. G.; Caramella, P. Tetrahedron 1999, 55, 10497.

(c) Quadrelli, P.; Campari, P.; Mella, M.; Caramella, P. Tetrahedron Lett. 2000, 41, 2019.

[31] Quadrelli, P.; Invernizzi, A. G.; Caramella, P. Tetrahedron Lett. 1996, 37, 1909.

[32] (a) Knight, G. T.; Pepper, B. Tetrahedron 1971, 27, 6201. (b) Knight, G. T.; Loadman, M. J. R. J. Chem. Soc., B 1971, 2107.

[33] Adam, W.; Krebs, O. Chem. Rev. 2003, 103, 4131.

[34] (a) Corrie, J. E. T.; Kirby, G. W.; Mackinnon, J. W. M. J. Chem. Soc., Perkin Trans. 1 1985, 883.

(b) Kirby, G. W.; McGuigan, H.; McLean, D. J. J. Chem. Soc., Perkin Trans. 1 1985, 1961.

[35] Kirby, G. W. Chem. Soc. Rev. 1977, 6, 12.

[36] Keck, G. E.; Webb, R. R.; Yates, J. B. Tetrahedron 1981, 37, 4007.

[37] Ensley, H. E.; Mahadevan, S. Tetrahedron Lett. 1989, 30, 3255.

[38] (a) Schollkopf, U; Tonne, P.; Schafer, H.; Markusch, P. Ann. Chem. 1969, $722,45$.

(b) Schollkopf, U; Tonne, P. Ann. Chem. 1971, 753, 135.

[39] (a) O'Bannon, P. E.; Dailey, W. P. Tetrahedron Lett. 1988, 29, 987. (b) O'Bannon, P. E.; Dailey, W. P. Tetrahedron Lett. 1988, 29, 5719.

[40] Quadrelli, P.; Mella, M.; Caramella, P. Tetrahedron Lett. 1998, 39 , 3233.

[41] Quadrelli, P.; Mella, M.; Invernizzi, A. G.; Caramella, P. Tetrahedron 1999, 55, 10497.

[42] Adam, W.; Bottke, N.; Krebs, O.; Saha-Moèller, C. R. Eur. J. Org. Chem. 1999, 1963.

[43] Iwasa, S.; Fakhruddin, A.; Tajima, K.; Nishiyama, H. Tetrahedron Lett. 2004, 45, 9323.

[44] Malkov, A. V. Atkinson, D.; Kabeshov, M. A.; Edgar, M. Adv. Synth. Catal. 2011, 353, 3347.

[45] Read de Alaniz, J.; Frazier, C. P.; Engelking, J. R. J. Am. Chem. Soc. 2011, 133, 10430.

[46] Read de Alaniz, J.; Frazier, C. P.; Engelking, J. R. Org. Lett. 2012, 14,3620 .

[47] Lu, C-D.; Tusun, X. Synlett 2012, 1801

[48] (a) Johannsen, M.; Jùrgensen, K. A. J. Org. Chem. 1994, 59, 214. (b) Johannsen, M.; Jùrgensen, K. A. J. Org. Chem. 1995, 60, 5979. (c) Srivastava, R. S.; Nicholas, K. M. J. Am. Chem. Soc. 1996, 118, 3311 .

(d) Srivastava, R. S.; Nicholas, K. M. J. Am. Chem. Soc. 1997, 119, 3302.

(e) Srivastava, R. S.; Nicholas, K. M. J. Org. Chem. 1994, 59, 5365 .

(f) Mùller, E. R.; Jùrgensen, K. A. J. Am. Chem. Soc. 1993, 115, 
11814.

(g) Ho, C.-M.; Lau, T.-C. Chem. Commun. 2000, 24, 859.

(h) Hogan, G. A.; Gallo, A. A.; Nicholas, K. M.; Srivastava, R. S. Tetrahedron Lett. 2002, 43, 9505.

(i) Srivastava, R. S. Tetrahedron Lett. 2003, 44, 3271.

[49] Sharpless, K. B. J. Am. Chem. Soc. 1978, 100, 7061.

[50] (a) Cenini, S. Ragaini, F.; Tollari, S.; Paone D. J. Am. Chem. Soc. 1996, 118, 11964.

(b) Srivastava, R. S.; Kolel-Veetil, M. K.; Nicholas, K. M. Tetrahedron Lett. 2002, 43, 931.

[51] Merişor, E.; Conrad, J.; Klaiber, I.; Mika, S.; Beifuss, U. Angew. Chem., Int. Ed. 2007, 46, 3353.

[52] Merişor, E.; Conrad, J.; Klaiber, I.; Mika, S.; Beifuss, U. Synlett 2010, 1766.

[53] Roberts, J. S.; Motherwell, W. B. J. Chem. Soc., Chem. Commun. $1972,328$.

[54] Solomon, D. H.; Moad, G.; Rizzardo, E. Tetrahedron Lett. 1981, 22, 1165.

[55] Haszeldine, R. N.; Barlow, M. G.; Murria, K. W. J. Chem. Soc., Perkin Trans. 1 1980, 1960.

[56] Schenk, C.; De Boer, T. J. Tetrahedron 1979, 35, 147.

[57] Miller, M. J.; Yang, B. Tetrahedron Lett. 2010, 51, 328.

[58] Keck, G. E.; Webb, R. R. J. Am. Chem. Soc. 1981, 103, 3174.

[59] (a) Goodwin, S.; Shoolery, J. N.; Horning, E. C. J. Am. Chem. Soc. 1959, $81,3736$.

(b) Okamoto, T.; Torii, A.; Isogai, Y. Chem. Pharm. Bull. 1968, 16, 1860 .

(c) Wildman, W. C.; Bailey, D. T. J. Org. Chem. 1968, 33, 3749.

(d) Wildman, W. C.; Bailey, D. T. J. Am. Chem. Soc. 1970, 92, 5538 .

[60] Martin, S. F.; Puckette, T. A.; Colapret, J. A. J. Org. Chem. 1979, 44, 3391.
[61] Wijnberg, J. B. P. A.; Speckamp, W. N. Tetrahedron 1978, 34, 2579.

[62] (a) Matsumura, Y.; Aoyagi, S.; Kibayashi, C. Org. Lett. 2003, 5, 3249.

(b) Matsumura, Y.; Aoyagi, S.; Kibayashi, C. Org. Lett. 2004, 6, 965.

[63] Clive, D. L. J.; Yu, M.; Wang, J.; Yeh, V. S. C.; Kang, S. Chem. Rev. $\mathbf{2 0 0 5}, 105,4483$

[64] Vasella, A.; Zhang, F. Helv. Chim. Acta 2007, 90, 2315.

[65] Vasella, A.; Zhang, F. Helv. Chim. Acta 2008, 91, 2351.

[66] Fokin, A. A.; Yurchenko, A. G.; Rodionov, V. N.; Gunchenko, P. A.; Yurchenko, R. I.; Reichenberg, A.; Wiesner, J.; Hintz, M.; Jomaa, H.; Schreiner, P. R. Org. Lett. 2007, 9, 4379.

[67] Haley, M. M.; Jeffrey, J. L.; McClintock, S. P. J. Org. Chem. 2008, $73,3288$.

[68] Murru, S.; Gallo, A. A.; Srivastava, R. S. J. Org. Chem. 2012, 77, 7119.

[69] Hamer, J.; Macaluso, A. Tetrahedron Lett. 1963, 4, 381.

[70] Seymour, C. A.; Greene, F. D. J. Org. Chem. 1982, 47, 5226.

[71] Davies, A. G.; Schiesser, C. H. Tetrahedron 1991, 47, 1707.

[72] Adam, W.; Krebs, O. J. Org. Chem. 2003, 68, 2420.

[73] (a) Leach, A. G.; Houk, K. N. Chem. Commun. 2002, 12, 1243. (b) Leach, A. G.; Houk, K. N. J. Am. Chem. Soc. 2002, 124, 14820.

[74] Leach, A. G.; Houk, K. N. J. Am Chem. Soc. 2003, 125, 13825.

[75] Lu, X. Org. Lett. 2004, 6, 2813.

[76] Adam, W.; Wirth, T. Acc. Chem. Res. 1999, 32, 703.

[77] Adam, W.; Bottke, N.; Krebs, O.; Engels, B. J. Am. Chem. Soc. 2001, 123, 5542.

[78] Adam, W.; Bottke, N.; Krebs, O. J. Am. Chem. Soc. 2000, 122, 6791.

[79] Kreâe, G.; Felber, H.; Ritter, A.; Ascherl, B.; Vasella, A. Recl. Trav. Chim. Pays-Bas 1986, 105, 295.

[80] Adam, W.; Krebs, O. J. Am. Chem. Soc. 2002, 124, 12938.

(Zhao, X.) 